\title{
Platinum Nanoparticles As A Therapeutic Agent Against Dextran Sodium Sulfate-Induced Colitis In Mice
}

This article was published in the following Dove Press journal: International Journal of Nanomedicine

\author{
Suqin Zhu ${ }^{1,2}$ \\ Mingyong Zeng ${ }^{2}$ \\ Guangxin Feng ${ }^{2}$ \\ Haohao Wu (iD ${ }^{2}$ \\ 'Institute of Nutrition and Health, \\ Qingdao University, Qingdao 26602I, \\ People's Republic of China; ${ }^{2}$ College of \\ Food Science and Engineering, Ocean \\ University of China, Qingdao, Shandong \\ Province, 266003, People's Republic of \\ China
}

Purpose: This study aimed to evaluate the anti-colitis potential of platinum nanoparticles (PtNPs).

Materials and methods: 5-, 30- and 70-nm PtNPs were administered to C57BL/6 mice once daily by intragastric gavage for $8 \mathrm{~d}$ during and after 5 - $d$ dextran sodium sulfate treatment.

Results: According to body weight change, stool blood and consistency, and colon length and histopathology, PtNPs size-dependently alleviated DSS-induced murine colitis. PtNPs enhanced gut-barrier function by upregulating the colonic expressions of heat-shock protein 25 and tight junction proteins. Based on colonic myeloperoxidase activity, colonic and peripheral levels of interleukin- 6 and tumor necrosis factor- $\alpha$, and peripheral counts of white blood cells, PtNPs attenuated colonic and systemic inflammation. By suppressing lipopolysaccharide-triggered production of proinflammatory mediators, including nitric oxide, tumor necrosis factor- $\alpha$ and interleukin-6, PtNPs exerted direct anti-inflammatory activities in RAW264.7 macrophages through a mechanism involving intracellular reactive oxygen species scavenging and Toll-like receptor $4 / \mathrm{NF}-\kappa \mathrm{B}$ signaling suppression. High-throughput 16S rRNA sequencing of fecal samples unveiled that PtNPs induced gut dysbiosis by unfavorably altering $\alpha$-diversity, Firmicutes/Bacteroidetes ratio, and richness of certain specific bacteria.

Conclusion: PtNPs are a promising anti-colitis agent, but may negatively impact gut-microbiota.

Keywords: platinum nanoparticles, ulcerative colitis, gut-microbiota, anti-inflammation, gut-barrier function

\section{Introduction}

Ulcerative colitis and Crohn's disease are known as inflammatory bowel diseases (IBD), which are featured by relapsing, incurable chronic inflammatory conditions of the colon. Up to $0.5 \%$ of the general population in Western countries are affected by IBD, and there are also more and more people becoming IBD patients in newly industrialized countries. ${ }^{1}$ IBD cause a significant impairment of the patient's healthrelated quality of life, leading to persistent diarrhea, abdominal pain, fatigue, and even colorectal cancer. ${ }^{2}$ The exact etiology of IBD is still unclear, but dysfunctional immune responses of the gut, in connection with an individual's genetic predisposition and many environmental triggers, is thought as the main culprit. ${ }^{3}$ Oxidative stress contributes a great part to immune activation and mucosal injury in the
Correspondence: Haohao Wu

College of Food Science and Engineering, Ocean University of China, 5 Yushan

Road, Qingdao, Shandong Province

266003, People's Republic of China

Tel +86-532-82032400

Fax +86-532-82032400

Email wuhaohao@ouc.edu.cn 
pathogenesis of IBD, and therefore antioxidants are being taken into account when designing novel anti-IBD therapies. $^{4}$

Nanomaterials have been found to possess efficient activities to catalyze a lot of chemical reactions. ${ }^{5-9}$ Metal nanoparticles are particularly good catalysts due to the high concentration of surface electrons. Platinum nanoparticles (PtNPs) can act as strong and stable mimetics of superoxide dismutase and catalase, ${ }^{10-12}$ and have been demonstrated to effectively attenuate oxidative stress-induced pulmonary inflammation and neurological impairment in mice. ${ }^{13-15}$ The oral administration of PtNPs causes little toxicity to mice and rats ${ }^{16,17}$ and, considering intestine as their first site of contact, oral PtNPs may serve as an effective antioxidative agent to attenuate intestinal injury and inflammation in IBD. PtNPs have been approved as a food additive by the Ministry of Health, Labor and Welfare of Japan, and there are commercially marketed foods supplemented with PtNPs, such as mineral water, candies, yogurt, and gum, in Japan. However, whether PtNPs can provide a therapeutic benefit against disease conditions in IBD patients is unknown. The present study assessed the protective potential of PtNPs with diameters of 5, 30 and $70 \mathrm{~nm}$ against dextran sodium sulphate (DSS)-induced colitis in C57BL/6 mice, when administered by oral gavage, and investigated the anti-inflammatory mechanism of PtNPs in a murine macrophage cell line, RAW264.7, when stimulated with lipopolysaccharide (LPS).

\section{Materials And Methods Materials}

Citrate-capped BioPure ${ }^{\mathrm{TM}}$ spherical PtNPs with the diameters of 5, 30 and $70 \mathrm{~nm}$ (Pt-5 nm, Pt-30 nm and Pt-70 $\mathrm{nm}$ ) were supplied as $1 \mathrm{mg} / \mathrm{mL}$ suspension in $2 \mathrm{mM}$ citrate buffer by nanoComposix, Inc. (San Diego, CA), and were guaranteed to be sterile and with an endotoxin level lower than $2.5 \mathrm{EU} / \mathrm{mL}$. The hydrodynamic and core sizes of PtNPs were measured with dynamic light scattering (DLS) on a Zetasizer Nano ZS instrument (Malvern Co., Herrenberg, Germany) at $25^{\circ} \mathrm{C}$, and with transmission electron microscopy (TEM) on a JEM 2100 instrument (JEOL Co., Tokyo, Japan) at $200 \mathrm{kV}$, respectively. DSS (36-50 kDa) was bought from Yisheng Biotechnology Co., Ltd (Shanghai, China). Phenylmethanesulfonyl fluoride (PMSF), methylthiazolyldiphenyl-tetrazolium bromide (MTT), the cocktail of protease and phosphatase inhibitors, LPS derived from Escherichia coli O55: B5, bovine serum albumin (BSA), radioimmunoprecipitation assay lysis buffer, and o-dianisidine dihydrochloride were provided by Sigma-Aldrich (Shanghai, China). Dulbecco's Modified Eagle medium (DMEM), the Pierce BCA protein assay kit, 2',7'-dichlorodihydrofluorescein (DCF) diacetate, kits for enzyme-linked immunosorbent assay (ELISA), Hank's Balanced Salt solution (HBSS), Dulbecco's Phosphate-Buffered Saline (DPBS), and the enhanced chemiluminescence (ECL) detection kit, were bought from ThermoFisher Scientific Co., Ltd. (Beijing, China). Fetal bovine serum (FBS) was provided by ExCell Bio (Shanghai, China). Mouse monoclonal anti-Toll-like receptor 4 (TLR4) antibody, Alexa Fluor ${ }^{\circledR}$ 647-conjugated mouse monoclonal anti-TLR4 antibody and its matched isotype control were provided by Santa Cruz Biotechnology (Beijing, China). The primary antibodies of zonula occludens-1 (ZO-1), nuclear factor kappa B $(\mathrm{NF}-\kappa \mathrm{B}) \mathrm{p} 65$, heat-shock protein 25 (Hsp25), $\beta$-actin (ab6276), occludin, claudin-4, and secondary anti-rabbit IgG, Alexa Fluor ${ }^{\circledR}$ 488-conjugated anti-mouse IgG antibodies and anti-rabbit IgG were provided by Abcam (Cambridge, MA). Rabbit monoclonal anti-inducible nitric oxide (NO) synthase (iNOS) antibody was purchased from Cell Signaling Technology (Shanghai, China).

\section{Animals}

Seven to eight weeks old male mice (C57BL/6) weighing $21.3 \pm 1.6 \mathrm{~g}$ were obtained from Lukang Pharmaceutical Co., Ltd (Jining, China). Mice were kept individually (one cage per mouse) at $20-24^{\circ} \mathrm{C}$ in an air-conditioned house with 55-65\% humidity under the 12-hr light: 12-hr dark condition. Animals were given a pelletized chow diet and deionized water ad libitum. All experiments were approved by the Animal Ethical and Welfare Committee of Ocean University of China, and were carried out ethically as per the guidelines of National Institutes of Health Guide for the Care and Use of Laboratory Animals.

\section{Protocol For Acute Colitis Induction And Treatment}

After a 3-d acclimation period, animals were randomly assigned to 5 groups ( $\mathrm{n}=8$ per group), comprising DSS control, normal control, DSS + Pt-5 nm, DSS + Pt-30 nm, and DSS + Pt-70 nm. The normal control mice received drinking water ad libitum for 8 days. The other groups received 3\% (w/v) DSS drinking water ad libitum for $5 \mathrm{~d}$ to induce acute colitis, and had free access to normal 
drinking water for the following $3 \mathrm{~d}$. During the $8-\mathrm{d}$ experimental period, all mice were administered by daily gastric intubations with $200 \mu \mathrm{L}$ of deionized water for the groups of DSS control and normal control or $2.8 \mu \mathrm{g} / \mathrm{mL}$ of the specified PtNPs for the platinum-treated groups. The dosage of PtNPs was approximately equivalent to $25 \mu \mathrm{g}$ platinum/kg bodyweight. The experimental plan is shown in Figure S1.

\section{Evaluation Of Acute Colitis}

Food consumption and body weight were recorded daily for each mouse, and clinical symptoms of colitis (i.e. fecal blood and stool consistency) for all mice were observed each day. ${ }^{18}$ The loss of body weight was rated as follows: 0 for less than $1 \% ; 1$ for $1-5 \%$; 2 for $5-10 \% ; 3$ for over $10 \%$. Fecal blood was rated as follows: 0 for no blood; 1 for appearance of blood around anus or in stool; 2 for severe bleeding. Stool consistency was rated as follows: 0 for normal; 1 for sticky/moist stool; 2 for soft stool; 3 for diarrhea. The scores of weight loss, fecal blood, and stool consistency were added up to yield the Disease Activity Index (DAI), which was used to evaluate the severity of colitis.

\section{Animal Sacrifice And Necropsy}

Fecal samples were collected at day 8 , and were kept at $-80^{\circ} \mathrm{C}$ before gut-microbiota profiling. After the mice were anesthetized via ether inhalation for $60 \mathrm{~s}$, the blood was collected from the orbital sinus following the procedure described by Parasuraman et al (2010). ${ }^{19}$ The animals were then immediately euthanized by cervical dislocation. Hematological analysis of blood samples collected in ethylenediamine tetra-acetic acid (EDTA)-containing tubes was performed on an XN-9000 automated hematology analyzer (Sysmex Co., Kobe, Japan). Blood samples collected in tubes without anticoagulant were allowed to clot for $0.5 \mathrm{hr}$, and serum for cytokine determination was then obtained by centrifugation (3000 rpm, $15 \mathrm{mins})$ of the clotted samples at $4^{\circ} \mathrm{C}$. After intact removal of the colon from abdominal cavity, colon length was measured from the ileocecal valve to the anus in a relaxed position without stretching, and for analysis of histopathological and biochemical parameters, the proximal part of colon was cut into several $1-\mathrm{cm}$ long pieces.

\section{Histopathological Examination}

A 1-cm segment of proximal colon was fixed with neutral formaldehyde solution, desiccated, embedded in paraffin, and sliced into 5- $\mu \mathrm{m}$ thickness pieces before a routine hematoxylin and eosin (H\&E) staining. Histologic observation was performed on a BX41 light microscope (Olympus Optical Co Ltd, Tokyo, Japan). Six fields from each sample were selected at random and imaged. A histologic scoring criteria of Laroui et al (2012) were used to rate the degree of mucosal damage. ${ }^{18}$ The histologic score for each animal was an average score of six fields.

\section{Myeloperoxidase (MPO) Assay}

Colonic MPO activity was determined following the procedure of Rodriguez-Palacios et al (2015). ${ }^{20}$ A preweighed segment of the proximal colon, which was kept at $-80^{\circ} \mathrm{C}$ for less than $48 \mathrm{hrs}$, was homogenized in 20 volumes of ice-cold buffer $\left(50 \mathrm{mM} \mathrm{K}_{2} \mathrm{HPO}_{4}\right.$ and $50 \mathrm{mM}$ $\left.\mathrm{KH}_{2} \mathrm{PO}_{4}, \mathrm{pH} 6.0\right)$ containing $0.5 \%(\mathrm{w} / \mathrm{v})$ cetyltrimethylammonium bromide on a Bioprep-6 Homogenizer (Aosheng Co., Shanghai, China). After centrifugation (10,000 g, 5 mins) of the homogenates at $4^{\circ} \mathrm{C}$, the supernatants were added to the wells in a volume of $10 \mu \mathrm{L}$ per well to a pre-chilled 96-well plate, and then, the active substrate solution containing $0.001 \% \mathrm{H}_{2} \mathrm{O}_{2}$ and o-dianisidine hydrochloride $(0.2 \mathrm{~g} / \mathrm{L})$ was loaded to the wells in a volume of $200 \mu \mathrm{L}$ per well. Immediately, the absorbance was read at $450 \mathrm{~nm}$ every $30 \mathrm{~s}$ for 5 mins on a Synergy H4 microplate reader (Bio-Tek Instruments Inc., Winooski, USA) at $28^{\circ} \mathrm{C}$. The MPO activity was defined as: 1 unit $=\Delta \mathrm{A} 450$ $\div 0.5 \div 0.0113 \div 0.05$; where $\Delta \mathrm{A} 450$ was the mean value of $\Delta \mathrm{A} 450_{(\mathrm{t} 30-\mathrm{t} 0)}$ (the change in absorbance during 0-30 s) and $\Delta \mathrm{A}_{450_{(\mathrm{t} 60-\mathrm{t} 30)}}$ (the change in absorbance during 0-30 s); 0.5 was the time interval (i.e. $0.5 \mathrm{~min}$ ); 0.0113 was the MPO constant; 0.05 was the dilution factor (i.e. $50 \mathrm{mg}$ sample: $1 \mathrm{~mL}$ buffer).

\section{Measurement Of Cytokines, Hsp25 And Tight Junction Proteins}

A pre-weighed segment of the proximal colon was homogenized in 25 volumes of radioimmunoprecipitation assay lysis buffer containing a cocktail of protease and phosphatase inhibitors and PMSF (1 $\mathrm{mM})$ on a Bioprep-6 Homogenizer. After centrifugation $(10,000 \mathrm{~g}, 5 \mathrm{mins}$, $4^{\circ} \mathrm{C}$ ) of the homogenate, the supernatant was obtained as the tissue extract. Tumor necrosis factor- $\alpha$ (TNF- $\alpha)$ and interleukin-6 (IL-6) in tissue extract and serum were quantified by using ELISA kits according to the instructions of the manufacturer. Claudin-4, ZO-1, occludin and Hsp25 in tissue extracts were determined with Western blotting. 


\section{Immunofluorescence}

After formalin fixation and paraffin embedment, colonic samples were sliced into $4-\mu \mathrm{m}$ thickness sections, and then, the sections were air dried overnight, deparaffinized in xylene and rehydrated, before antigen retrieval and blocking with 5\% BSA. Following an incubation with the antibodies for claudin-4, ZO-1 and occludin overnight at $4^{\circ} \mathrm{C}$, the sections were further incubated with an Alexa Fluor $^{\circledR}$ 488-labeled secondary antibody in dark at room temperature for 60 mins. Specimens were kept in mounting medium until being imaged by an Eclipse Ti-SR fluorescence microscope (Nikon Co., Tokyo, Japan).

\section{Gut-Microbiota Profiling}

Fecal samples were randomly chosen from the groups of DSS control $(n=5)$, normal control $(n=5)$, and DSS + Pt-5nm $(n=5)$ for gut-microbiota analysis. Total bacterial DNA was extracted from the fecal materials with the QIAamp DNA Stool Mini Kit (Qiagen Co., Hilden, Germany) according to the instructions of the manufacturer. The $1 \%$ agarose gel electrophoresis was used to measure the purity, concentration, integrity and fragment size of the extracted DNA. The primers used for amplifying the hypervariable regions V4 to V5 of 16S rDNA gene were 515F (i.e. 5'-GTGYCAGCMGCCGCGGTAA-3') and 907R (i.e. 5'-CCYCAATTCMTTTRAGTTT-3'). An Illumina HiSeq2500 high-throughput sequencer (Illumina, San Diego, USA) was used for the 16S rRNA tag-encoded high-throughput sequencing. The paired-end reads were assigned into different samples on the basis of their unique barcodes and truncated by removal of barcodes and primer sequences. The FLASH algorithm was used to merge these paired-end reads. Quality filtering of the raw sequencing data was carried out to get high-quality clean tags, which were subsequently analyzed using the QIIME (Quantitative Insights Into Microbial Ecology) software with default settings. A set of sequences at a similar level of $97 \%$ was grouped into one Operational Taxonomic Unit (OTU) by the UPARSE pipeline, and by using Mothur as the assigner algorithm and Silva as the reference database, one sequence was chosen as the representative for each OUT to annotate taxonomic information. The chaol metric and principal-component analysis of unweighted unique fraction (UniFrac) were computed using QIIME software. The linear discriminant analysis (LDA) effect size (LEfSe) algorithm was employed to compare taxa among the groups.

\section{Cell Viability Assay}

Mouse macrophage RAW264.7 cells were purchased from the Cell Bank of the Chinese Academy of Sciences (Shanghai, China). They were routinely maintained in a $5 \% \mathrm{CO}_{2}$ atmosphere in a $37^{\circ} \mathrm{C}$ incubator in DMEM supplemented with $10 \%$ FBS. To assay cellular toxicity, cells seeded at $1 \times 10^{5}$ cells/well were cultured for $24 \mathrm{hrs}$ in a 96-well plate, and were then treated with or without $1.25 \mu \mathrm{g} / \mathrm{mL}$ PtNPs in complete media for $24 \mathrm{hrs}$. The MTT assay was then used to measure cell viability. Cells were incubated with MTT $(0.5 \mathrm{~g} / \mathrm{L}$ in fresh medium $)$ for $4 \mathrm{hrs}$, followed by dissolving of the resulted formazan crystals with DMSO, and the absorbance at $570 \mathrm{~nm}$ was then read on a Synergy H4 microplate reader.

\section{Measurement Of Cellular Production Of NO And Inflammatory Cytokines}

To evaluate the production of $\mathrm{NO}$ and cytokines, RAW264.7 cells seeded at $5 \times 10^{5}$ cells/well were cultured for $24 \mathrm{hrs}$ in a 48-well plate, and were then treated with or without $1.25 \mu \mathrm{g} / \mathrm{mL}$ PtNPs in complete media for $5 \mathrm{hrs}$. After rinsing with DPBS for 3 times, cells were treated with or without LPS $(1 \mu \mathrm{g} / \mathrm{mL}$ in complete media) for $20 \mathrm{hrs}$. The culture fluids were then harvested and prior to analysis, were stored at $-80^{\circ} \mathrm{C}$.

To assay NO production, nitrite concentrations in the culture fluids were measured with Griess reagent $(5 \%$ phosphoric acid, $0.1 \% \mathrm{~N}-1$-naphthyl ethylenediamine, and $1 \%$ sulphanilamide). Briefly, $100 \mu \mathrm{L}$ of the culture fluid was mixed with an equal volume of freshly prepared Griess reagent in a 96-well plate for about 10 mins, after which the absorbance at $540 \mathrm{~nm}$ was read on a Synergy H4 microplate reader. A sodium nitrite standard curve was used to calculate the nitrite concentration. The levels of TNF- $\alpha$ and IL- 6 in the culture fluids were assayed by using ELISA kits according to the instructions of the manufacturer.

\section{Intracellular Reactive Oxygen Species (ROS) Assay}

RAW264.7 cells seeded at $5 \times 10^{5}$ cells/well were cultured for $48 \mathrm{hrs}$ in a 48 -well plate, and were then treated with or without $1.25 \mu \mathrm{g} / \mathrm{mL}$ PtNPs in complete media for $5 \mathrm{hrs}$. After rinsing with HBSS for 3 times, cells were treated with or without LPS ( $1 \mu \mathrm{g} / \mathrm{mL}$ in complete media) for $1 \mathrm{hr}$, and were then incubated with $20 \mu \mathrm{M}$ of DCF diacetate for $0.5 \mathrm{hr}$ to allow dye diffusion into the cells, followed by 
rinsing with DPBS for two times. The fluorescence of DCF was read on a Synergy H4 microplate reader at $535 \mathrm{~nm}$ (emission) and $485 \mathrm{~nm}$ (excitation).

\section{Measurement Of Cellular TLR4, NF-kB P65 And iNOS}

TLR4 protein levels in cell lysate and on the cell surface were measured using Western blotting and flow cytometry, respectively. For these experiments, cells were seeded at a density of $1.0 \times 10^{6}$ cells/well in 6 -well plates and cultured for $48 \mathrm{hrs}$, and were then treated with or without $1.25 \mu \mathrm{g} / \mathrm{mL}$ PtNPs in complete media for $5 \mathrm{hrs}$, followed by rinsing with DPBS for 3 times. The single-cell suspension for flow cytometric analysis was prepared by detaching cells with EDTA and exposing cells to Alexa Fluor ${ }^{\circledR}$ 647-conjugated anti-TLR4 antibody or its matched isotype control on ice for $0.5 \mathrm{hr}$, and was analyzed on a BD Accuri C6 plus instrument (BD Biosciences, Shanghai, China). Cells were lysed by radioimmunoprecipitation assay lysis buffer supplemented with a cocktail of protease and phosphatase inhibitors and PMSF ( $1 \mathrm{mM})$ for $0.5 \mathrm{hr}$ at $4^{\circ} \mathrm{C}$, followed by centrifugation at $13,000 \mathrm{rpm}$ at $4^{\circ} \mathrm{C}$ for 15 mins, and the supernatants were then harvested as cell lysate samples for Western blotting analysis.

To assay the expression levels of NF- $\kappa$ B p65 and iNOS, cells were seeded at a density of $1.0 \times 10^{6}$ cells/ well in 6-well plates and cultured for $48 \mathrm{hrs}$. After an incubation with or without PtNPs $(1.25 \mu \mathrm{g} / \mathrm{mL})$ for $5 \mathrm{hrs}$, cells were rinsed three times with DPBS and stimulated with or without LPS $(1 \mu \mathrm{g} / \mathrm{mL})$ for $1 \mathrm{hr}(\mathrm{NF}-\kappa \mathrm{B}$ p65) or $24 \mathrm{hrs}$ (iNOS). Cells were then lysed in radioimmunoprecipitation assay lysis buffer supplemented with a cocktail of protease and phosphatase inhibitors and PMSF (1 mM) for $0.5 \mathrm{hr}$ at $4^{\circ} \mathrm{C}$, followed by centrifugation at $13,000 \mathrm{rpm}$ at $4{ }^{\circ} \mathrm{C}$ for 15 mins. The supernatant was harvested as cell lysates for Western blotting analysis.

\section{Western Blotting}

Protein concentrations in tissue extracts and cell lysates were determined by the Pierce BCA assay according to the instructions of the manufacturer. The cell lysate samples were boiled in sodium dodecyl sulphate-loading buffer, separated with sodium dodecyl sulphate-polyacrylamide gel electrophoresis, and then blotted onto a PVDF membrane. After blocking in TBST [10 $\mathrm{mM}$ tris $(\mathrm{pH} 7.5)$, $150 \mathrm{mM} \mathrm{NaCl}$, and $0.05 \%$ Tween 20] supplemented with $5 \%$ fat-free dried milk at room temperature for $1 \mathrm{hr}$, the membrane was incubated overnight at $4{ }^{\circ} \mathrm{C}$ with antibodies for $\beta$-actin, TLR4, iNOS, NF-кB p65, Claudin-4, Hsp-25, Occludin, or ZO-1. After incubation with HRP-conjugated secondary antibodies for $1 \mathrm{hr}$, the immune complexes were washed 3 times with TBST, followed by visualization with an ECL Western blotting detection kit on a 5200 Multi Luminescent image analyzer (Tanon Science and Technology Co., Ltd., Shanghai, China).

\section{Statistical Analysis}

OriginPro 7.0 software (OriginLab Co., Northampton, USA) and SPSS 19.0 software (SPSS Co., Chicago, USA) was used to perform statistical analysis. The mean difference was compared with two-paired Student's t-test, oneway analysis of variance followed by post-hoc Turkey's honestly significantly difference test, or Wilcoxon signedrank test. Differences with probability $(P)$ value $<0.05$ were considered significant.

\section{Results}

\section{Particle Characterization}

As examined by TEM (Figure 1), the core sizes of Pt-5 nm, Pt-30 nm and Pt-70 nm were in accordance with those declared by the manufacturer. Table 1 summarizes results of the DLS measurements of PtNPs. When dispersed in water, Pt-5nm gave a hydrodynamic size as large as $89.5 \mathrm{~nm}$, suggesting aggregation of the particles; nevertheless, a much smaller hydrodynamic size of $14.6 \mathrm{~nm}$ was observed for Pt-5nm dispersed in 10\% FBS-supplemented DMEM, validating the colloidal stabilization effect associated to protein corona formation around nanoparticles in biological media. Pt-30 nm and Pt-70 nm displayed slightly larger hydrodynamic sizes in 10\% FBS-supplemented DMEM than in water, indicating their limited aggregation under physiological salt conditions.

\section{Orally Administered PtNPs Ameliorated DSS-Induced Colitis}

To assess their protecting potential against acute colitis, PtNPs were administered to mice for $8 \mathrm{~d}$ by intragastric gavage during and after DSS exposure for $5 \mathrm{~d}$ (Figure $\mathrm{S} 1$ ). The kinetics of body weight (Figure 2A) revealed significant body weight loss of the DSS control group in comparison with the normal control group from day $1(P<0.01)$. Intragastric treatments with Pt-5 nm, Pt-30 nm and Pt-70 nm significantly attenuated the DSS-induced weight loss from day 1 , day 1 , and day 2 , respectively $(P<0.01)$, 


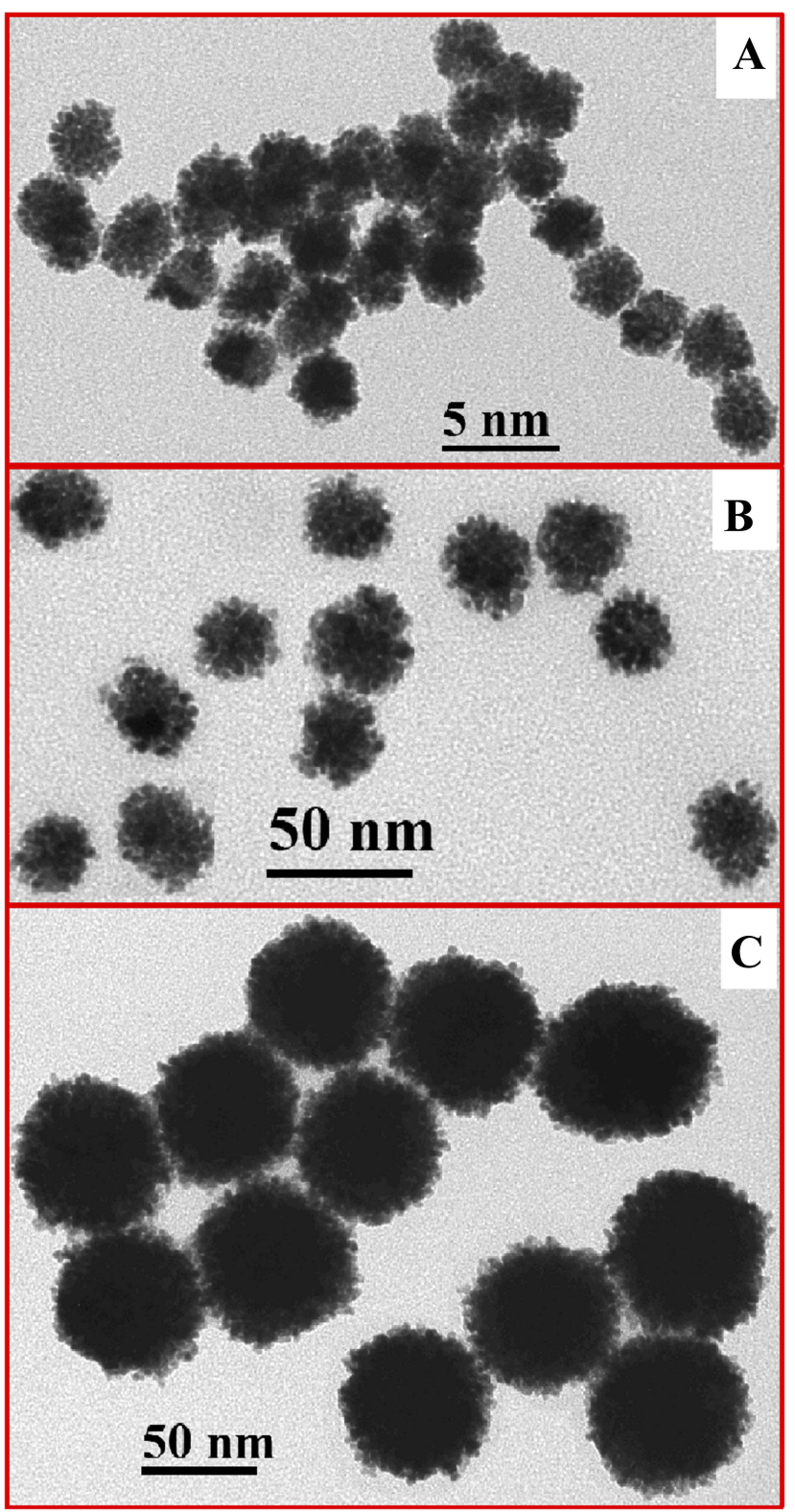

Figure I Transmission electron microscopy (TEM) images of platinum nanoparticles (PtNPs): (A) Pt-5nm; (B) Pt-30nm; (C) Pt-70nm.

with the protecting activities of PtNPs following a sizedependent order, that is, Pt-5 nm $>$ Pt-30 nm $>$ Pt-70 nm, from day $6(P<0.01)$.

As indicated by the DAI values (Figure 2B), free drinking of DSS caused remarkable pathological conditions (i.e. weight loss, fecal blood, and loose stool) in mice of the DSS control group, in comparison with the normal control group, from day $1(P<0.01)$. Oral administration of PtNPs effectively improved these pathological conditions from day $2(P<0.01)$, with the protecting effects of PtNPs following a size-dependent order, namely, Pt-5 nm $>$ Pt-30 nm $>$ Pt-70 nm, from day $7(P<0.01)$.
Table I The Hydrodynamic Sizes Of Platinum Nanoparticles (ptnps) Dispersed In Water And 10\% Fetal Bovine Serum (FBS)-Supplemented Dulbecco's Modified Eagle Medium (DMEM)

\begin{tabular}{|l|l|l|}
\hline \multirow{2}{*}{ PtNPs } & \multicolumn{2}{|l|}{$\begin{array}{l}\text { Size By Dynamic Light Scattering } \\
\text { Intensity-Weighted Peak (nm) }\end{array}$} \\
\cline { 2 - 3 } & Water & DMEM+FBS \\
\hline Pt-5nm & $89.5 \pm 4.2$ & $14.6 \pm 2.2$ \\
Pt-30nm & $37.8 \pm 1.0$ & $91.3 \pm 2.8$ \\
Pt-70nm & $105.7 \pm 6.0$ & $155.8 \pm 3.7$ \\
\hline
\end{tabular}

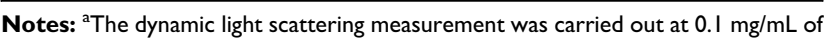
PtNPs. Data are shown as mean \pm standard deviations of 3 measurements.

The length of colon is a classical marker of intestinal pathology in acute colitis. As shown in Figure 2C and D, the DSS control had remarkably shorter colons $(6.3 \pm 0.6 \mathrm{~cm})$ than the normal control that had $8.7 \pm 1.1 \mathrm{~cm}$ long colons $(P<0.01)$, suggesting a marked intestinal pathological condition induced by the 5-d drinking of DSS. The DSS-induced colon shortening was attenuated by the orally administered PtNPs $(P<0.05)$, with $P t-70 \mathrm{~nm}$ showing a significantly lower amending effect than Pt-5 nm $(P<0.05)$.

Histological evaluation of H\&E-stained colonic tissue (Figure 2E) revealed characteristic pathological features of colitis, such as disordered and thinned structure of mucosa, and massive invasion of submucosa and mucosa by inflammatory cells, in the DSS control-treated mice. The oral administration of PtNPs protected against the DSS-induced histological lesions (Figure 2E), and based on the results of histological scoring (Figure 2F), the protecting efficiencies of PtNPs followed a size-dependent order, i.e. Pt-5 nm $>$ Pt-30 nm $>$ Pt-70 nm $(P<0.05)$.

According to the above results of weight loss, DAI, colon shortening and colon histopathology, PtNPs, apparently, have an anti-colitis potential that is dependent on their sizes. In the following parts, the gut barrier-protecting, anti-inflammatory and gut-microbiota modulating potentials of PtNPs will be examined to elucidate the anti-colitis mechanism.

\section{Oral Administration Of PtNPs Protected Gut-Barrier Function In Acute Colitis}

The leakage of intestinal epithelial barrier is a critical step to initiate and propagate intestinal inflammation in IBD. ${ }^{21}$ The expressions of tight junction proteins in colon were measured by using Western blotting (Figure 3A and B). In comparison with the normal control, the DSS control-treated animals displayed remarkably reduced levels of colonic claudin- 4 , 

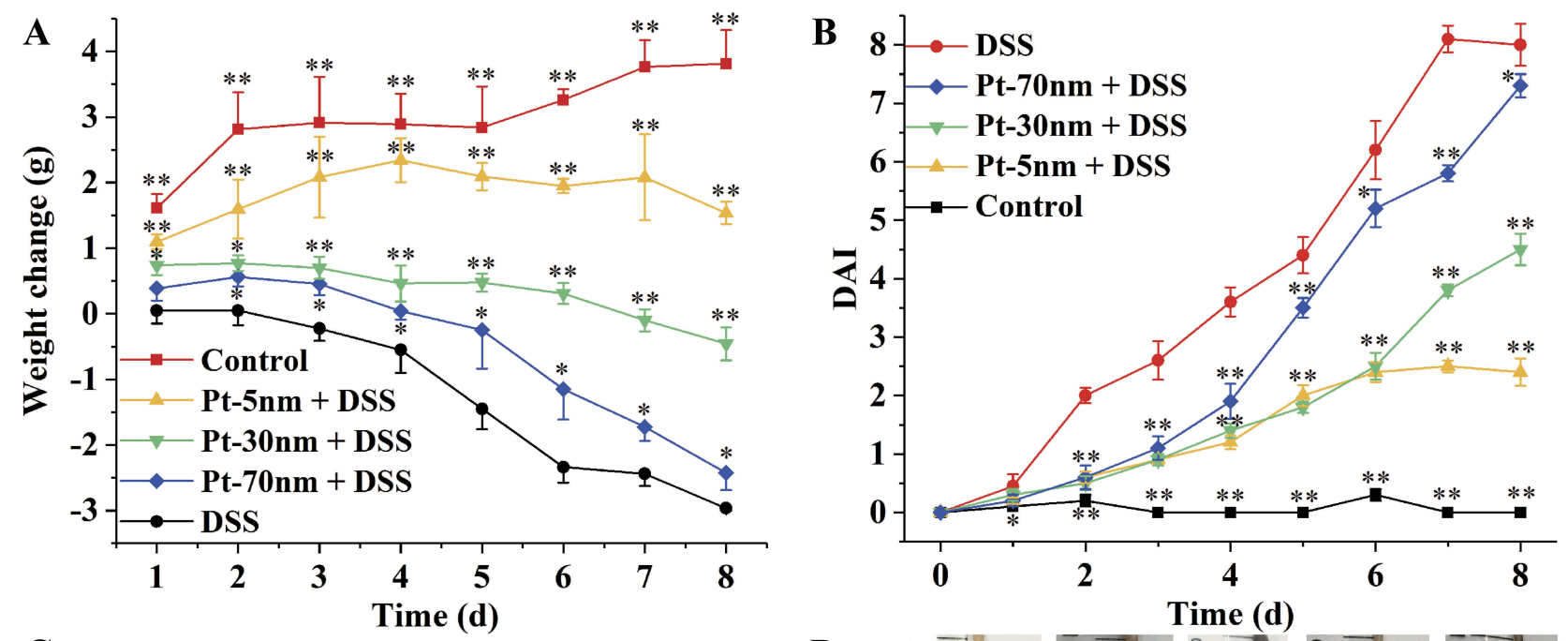

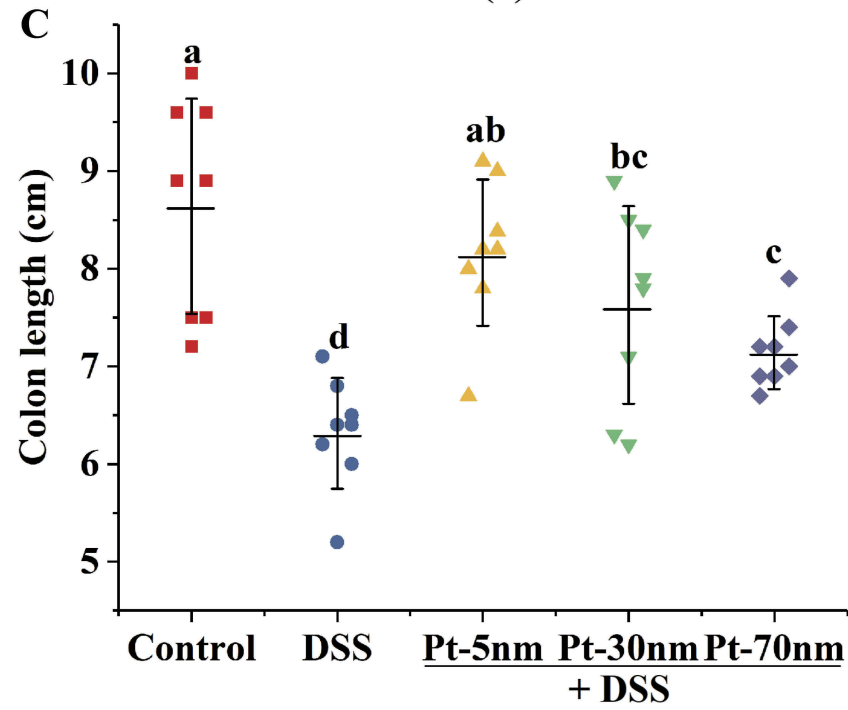

D

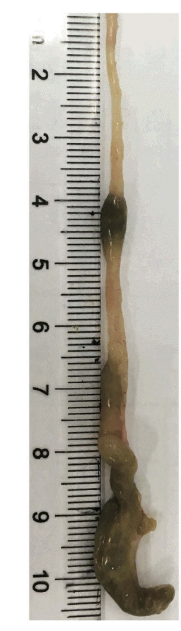

Control

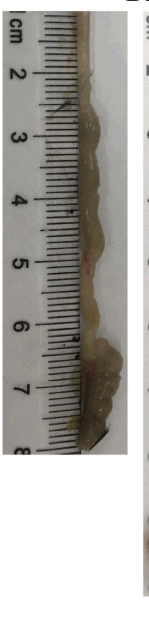

Time (d)

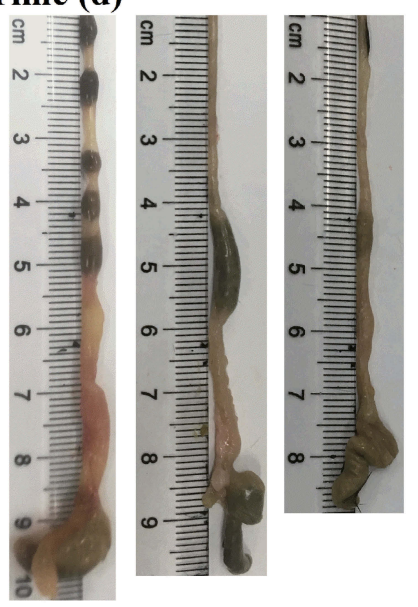

E

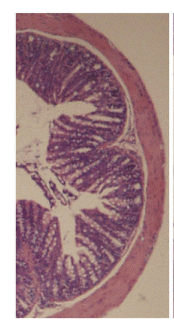

Control

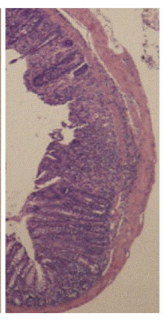

DSS

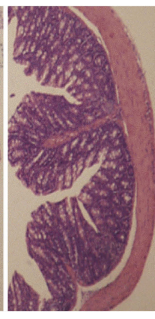

Pt-5nm

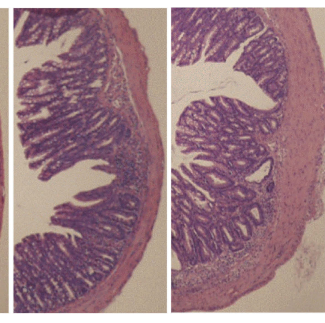

+ DSS
F 10

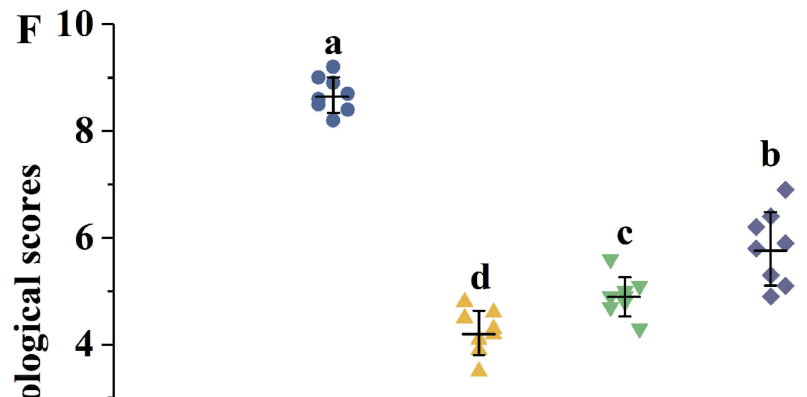

Figure 2 The DSS-induced clinical symptoms: (A) body weight kinetics; (B) Disease Activity Index (DAI); (C) statistical analysis and (D) representative images of colon length; (E) typical images and $(\mathbf{F})$ histopathological scores of colonic tissues. Data in $(\mathbf{A})$ and $(\mathbf{B})$ are presented as mean \pm standard deviations with significant differences versus the DSS control represented by asterisks (**P $<0.01$, $* \mathrm{P}<0.05$, two-paired Student's t-test). Each point in $(\mathbf{C})$ and $(\mathbf{F})$ represents the result of one animal, with significance labeled by different lower-case letters $(P<0.05$, one-way analysis of variance with Turkey's honest significant difference test). 


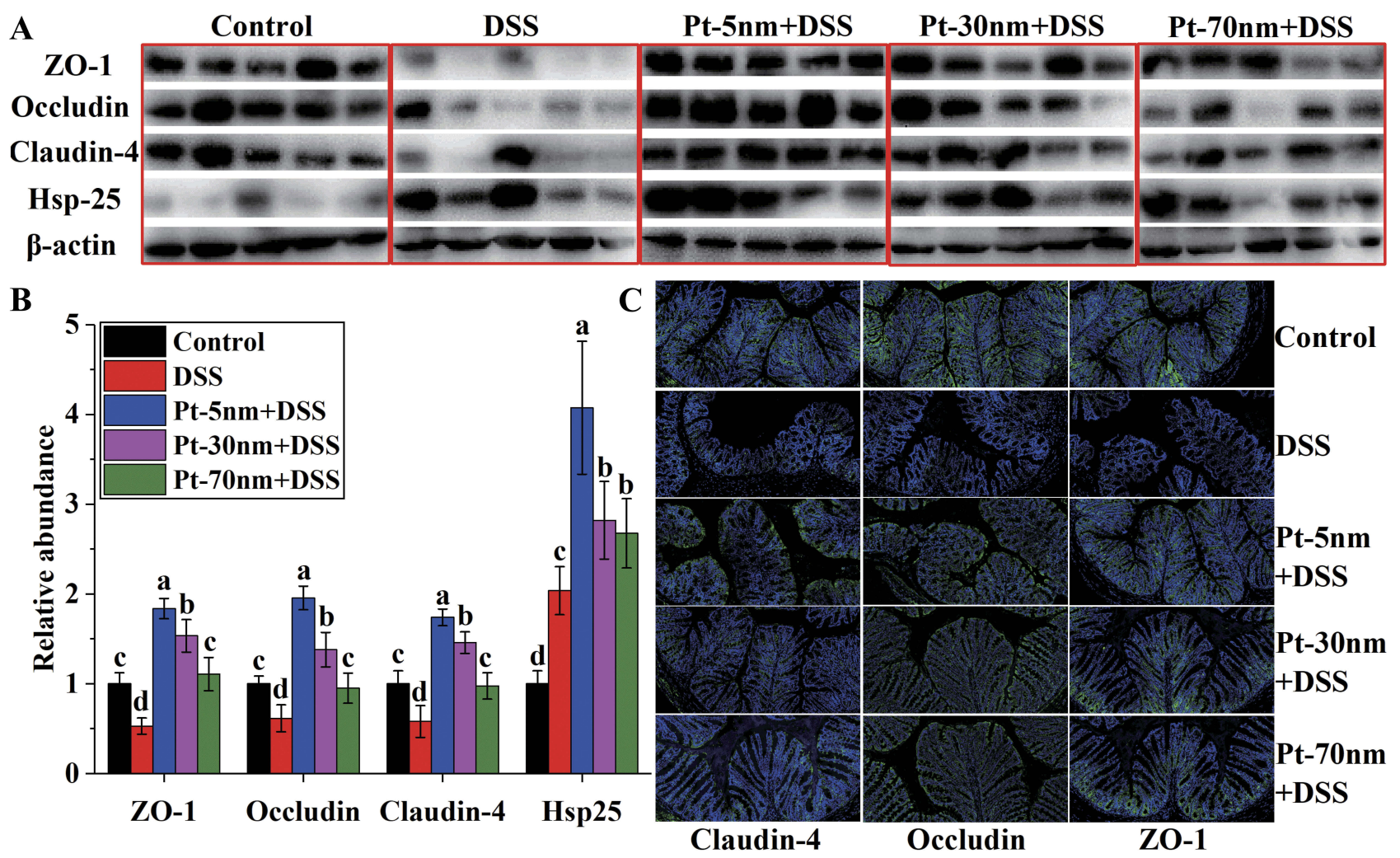

Figure 3 Immunological analysis of tight junction proteins and Hsp25 expressed in colon: (A) images and (B) densitometry of Western blotting analysis; (C) immunolocalization of tight junction proteins by immunofluorescence microscopy (200x). Blots in (A) are representative of at least 5 animals in each group, and experiments were performed for 3 times. Densitometry results in (B) are given in relation to normal controls that were set to be 1.0 . Significance is labeled by different lower-case letters $(P<0.05$, one-way analysis of variance with Turkey's honest significant difference test).

occludin and ZO-1, while orally administered PtNPs effectively suppressed the DSS-induced loss of these tight junction proteins in colon $(P<0.05)$. The tight junctionprotecting efficacies of PtNPs followed a size-dependent manner, i.e. Pt-5 nm $>$ Pt-30 nm $>$ Pt-70 nm $(P<0.05)$, which aligned well with the anti-colitis activities of PtNPs. The claudin-4, occludin and ZO-1 levels in colonic samples from the groups of DSS + Pt-5 nm and DSS + Pt-30 nm were even higher than those in the normal control group, suggesting a role of PtNPs to induce the expressions of tight junction proteins in colon. The results of immunofluorescence microscopy (Figure 3C) also revealed the preservation of ZO-1, claudin-4, and occludin at intestinal epithelial tight junctions by oral treatments with PtNPs.

Heat-shock proteins are a kind of stress-responsive chaperones that can protect mammalian cells against adverse conditions. $^{22}$ According to the Western blotting analysis (Figure $3 \mathrm{~A}$ and $\mathrm{B}$ ), the DSS control group displayed a significantly greater level of colonic Hsp25 than the normal control $(P<0.05)$, validating the stress-responsive expression of cytoprotective heat-shock proteins during the pathogenesis of IBD. ${ }^{23}$ Oral administration of PtNPs greatly increased the expression of Hsp25 in DSS-induced colitis mice $(P<0.05)$, suggesting a cytoprotective role of PtNPs in the maintenance of gut-barrier function.

\section{Attenuation Of Colonic And Systemic Inflammation By Oral Treatments With PtNPs In DSS-Administered Mice}

MPO is the most abundant protein in neutrophils, and the presence of its activity in gut tissues has been widely utilized to estimate intestinal infiltration of inflammatory cells. ${ }^{20}$ As shown in Figure 4A, DSS exposure remarkably increased the colonic MPO activity in mice of the DSS control group, in comparison with the normal control group $(P<0.05)$. Orally administered PtNPs repressed most of the MPO activity resulted from DSS exposure $(P<0.05)$; nevertheless, their protecting activities displayed no significant size dependence.

Levels of the pro-inflammatory cytokines of IL-6 and TNF- $\alpha$ in colonic and serum samples were measured to assess colonic and systemic inflammation (Figure 4B-E). DSS exposure induced a burst of both colonic and 


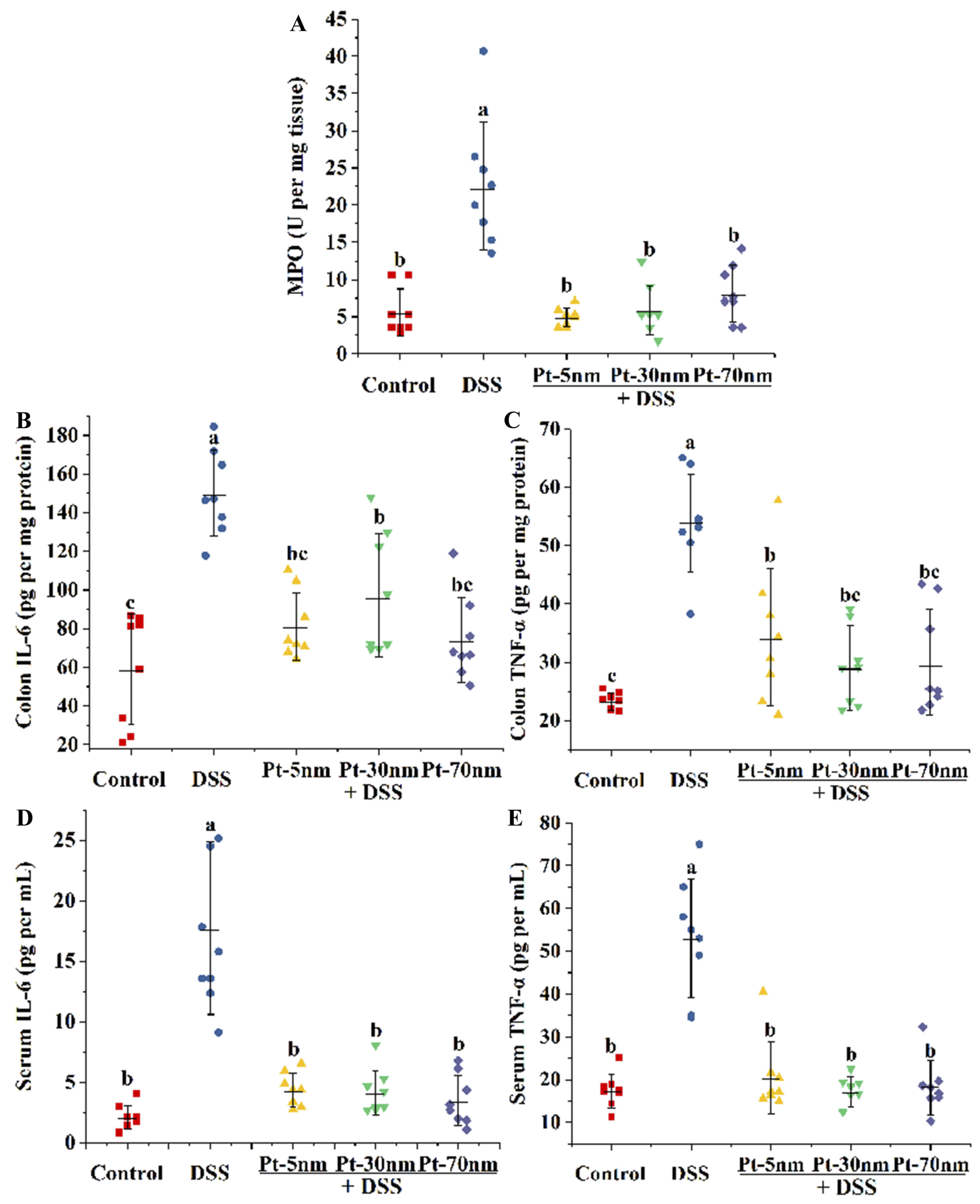

Figure 4 Biochemical parameters of acute colitis: (A) colonic MPO activity; the colonic levels of (B) IL-6 and (C) TNF- $\alpha$; the serum concentrations of (D) IL-6 and (E) TNF-a. Each point represents the result of one animal, with significance labeled by different lower-case letters $(P<0.05$, one-way analysis of variance with Turkey's honest significant difference test). 

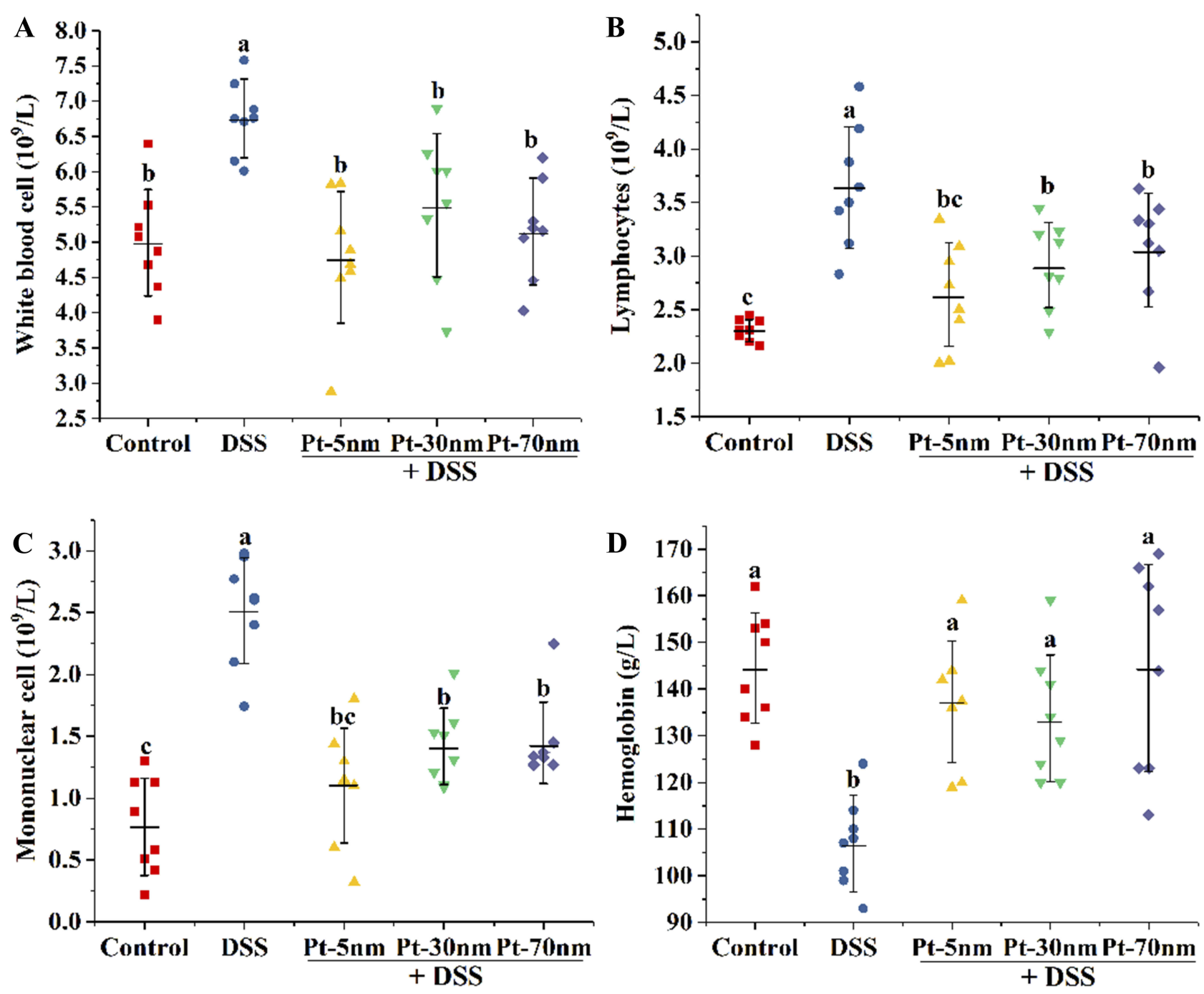

Figure 5 The peripheral hematological parameters: $(\mathbf{A})$ white blood cells, (B) lymphocytes, $(\mathbf{C})$ monocytes and (D) hemoglobin. Each point represents the result of one animal, with significance labeled by different lower-case letters $(P<0.05$, one-way analysis of variance with Turkey's honest significant difference test).

peripheral concentrations of IL-6 and TNF- $\alpha$ in the DSS control-treated mice, in comparison with the normal control-treated ones $(P<0.05)$. Orally administered PtNPs effectively attenuated the DSS-induced colonic and peripheral burst of these proinflammatory cytokines $(P<0.05)$, with their protecting activities showing no significant size dependence again.

Hematologic parameters have diagnostic importance in detecting inflammatory diseases, and an increased count of white blood cells is among the most widely used markers for systemic inflammation. DSS exposure gave rise to the counts of white blood cells as well as lymphocytes and monocytes, two main types of white blood cells, in the DSS control-treated mice, compared to the normal control-treated ones $(P<0.05)$ (Figure $5 \mathrm{~A}-\mathrm{C})$. In contrast, intragastric treatments with PtNPs well prevented the DSS-induced rise in the counts of white blood cells, lymphocytes, and monocytes $(P<0.05)$, with the protecting activities of PtNPs showing no significant size dependence (Figure 5A-C). Patients with chronic inflammatory diseases often suffer from a certain degree of anemia. ${ }^{24}$ In this study, the DSS control group had a significantly reduced level of hemoglobin compared to the normal control group $(P<0.05)$, while orally administered PtNPs effectively suppressed the DSS-induced decrease of hemoglobin $(P<0.05)$ in a non-size-dependent manner (Figure 5D).

The above results of biochemical and hematological tests revealed an evident attenuation of colonic and systemic inflammatory responses by oral administration of PtNPs in DSS-induced colitis. This observation might be 
explained by two possible mechanisms: (i) PtNPs prevented the leakage of intestinal epithelial barrier, thereby mitigating the initiation and propagation of intestinal inflammation; (ii) PtNPs could exert direct anti-inflammatory effects. PtNPs displayed non-size-dependent efficacies to attenuate colonic and systemic inflammation, which misaligned with their above-mentioned gut barrier-protecting activities, so the mechanism (i) cannot fully explain the anti-inflammatory activities of PtNPs in DSS-induced colitis mice. The direct anti-inflammatory properties of PtNPs will be examined below in mouse macrophage RAW264.7 cells.

\section{PtNPs Attenuated LPS-Induced Inflammatory Responses In RAW264.7 Cells}

To measure the highest non-cytotoxic doses of PtNPs in RAW264.7 cells, the MTT test was done after a 24-hr incubation with various concentrations of Pt-5 nm, Pt-30 $\mathrm{nm}$ and Pt-70 nm. At doses of $1.25 \mu \mathrm{g} / \mathrm{mL}$ or lower, PtNPs induced no significant loss of macrophage viability (Figure 6A). To examine the anti-inflammatory effects of PtNPs, cells were treated with the particles $(1.25 \mu \mathrm{g} / \mathrm{mL})$ for $5 \mathrm{hrs}$ before LPS stimulation for an additional $20 \mathrm{hrs}$. In the absence of PtNPs, LPS induced a remarkable production of NO, TNF- $\alpha$ and IL-6, as well as intracellular ROS according to the DCF fluorescence, by RAW264.7 macrophages (Figure 6B-E) $(P<0.001)$, while the pretreatments with PtNPs significantly attenuated these effects $(P<0.001)$, suggesting that PtNPs possess direct anti-inflammatory potential in gut inflammatory milieu. Pt-70 nm showed weaker activities in inhibiting the production of IL-6, TNF- $\alpha$ and intracellular ROS than Pt-5 $\mathrm{nm}$ and Pt-30 nm $(P<0.05)$. No size dependence was observed in the attenuation of LPS-induced NO production by PtNPs.

$\mathrm{NF}-\kappa \mathrm{B}$ is a central regulator of proinflammatory cytokine production in macrophages, and according to the Western blotting analysis of $\mathrm{NF}-\kappa \mathrm{B}$ p65 protein (Figure 7A), its activation by 1 -hr LPS stimulation in RAW264.7 cells was significantly inhibited by the pretreatments with PtNPs for $5 \mathrm{hrs}(P<0.01)$. Pt-70 nm caused a less extent of NF- $\kappa B$ inhibition than Pt-5 nm and Pt-30 nm $(P<0.01)$, and this was in good accordance with the above results of the production of IL-6 and TNF- $\alpha$ in Figure 6C and D. PtNPs thus seem to suppress inflammatory cytokine production through blocking $\mathrm{NF}-\kappa \mathrm{B}$ activation in mouse macrophages.
As shown in Figure 7B, a 24-hr LPS stimulation caused marked iNOS expression in RAW264.7 cells $(P<$ 0.001 , which was significantly attenuated by the 5 -h pretreatments with PtNPs $(P<0.05)$. PtNPs showed no size dependence to suppress the LPS-stimulated iNOS expression, which aligned well with the results of NO production (Figure 6B). These results imply that PtNPs down-regulated the LPS-induced burst of NO production by suppressing the iNOS expression in mouse macrophages.

Macrophages specifically recognize exogenous LPS via the TLR4 receptor complex on their cell surfaces. The TLR4 levels in whole-cell lysate and on the cell surface were determined by using Western blotting analysis (Figure 7C) and flow cytometry (Figure 7D), respectively. The 5-hr pretreatments with PtNPs caused an evident reduction of TLR4 both in whole cell lysate and on the cell surface of RAW264.7 cells $(P<0.01)$, implying that PtNPs desensitized macrophages to LPS.

PtNPs displayed no size dependence in TLR4 reduction, and this was in line with the above findings for $\mathrm{NO}$ production (Figure 6B) and iNOS expression (Figure 7B), suggesting that TLR4 reduction might explain the NO-mediated anti-inflammatory activities of PtNPs.

\section{Orally Administered PtNPs Altered Mouse Gut-Microbiota Profile}

Accumulating evidence suggests that commensal microbiota play a pivotal role in the initiation and pathogenesis of IBD. ${ }^{26}$ To investigate whether orally administered PtNPs could alter gut-microbiota profile, high-throughput sequencing of the 16S rRNA genes (hypervariable regions $\mathrm{V} 4$ to V5) from fecal material was performed to determine mouse gut-microbiota composition in the groups of DSS control, normal control and DSS + Pt-5 nm. In total, 6515 OTUs were obtained from 1,126,667 sequences of these fecal samples. To determine whether bacterial taxa in the samples were fully recovered, a rarefaction analysis (Figure S2) was carried out, and the rarefaction curves exhibited a plateau, indicating that the vast majority of bacterial species in our samples had been recovered.

The $\alpha$-diversity (i.e. within-sample species richness) was estimated using the chaol index (Figure 8A). A significantly reduced $\alpha$-diversity was observed in the DSS + Pt-5 nm group than the groups of DSS control $(P<0.01)$ and normal control $(P<0.01)$. The gut-microbiota $\alpha$-diversity in DSS control animals was at a similar level to that in normal control ones. These results suggest that 
A

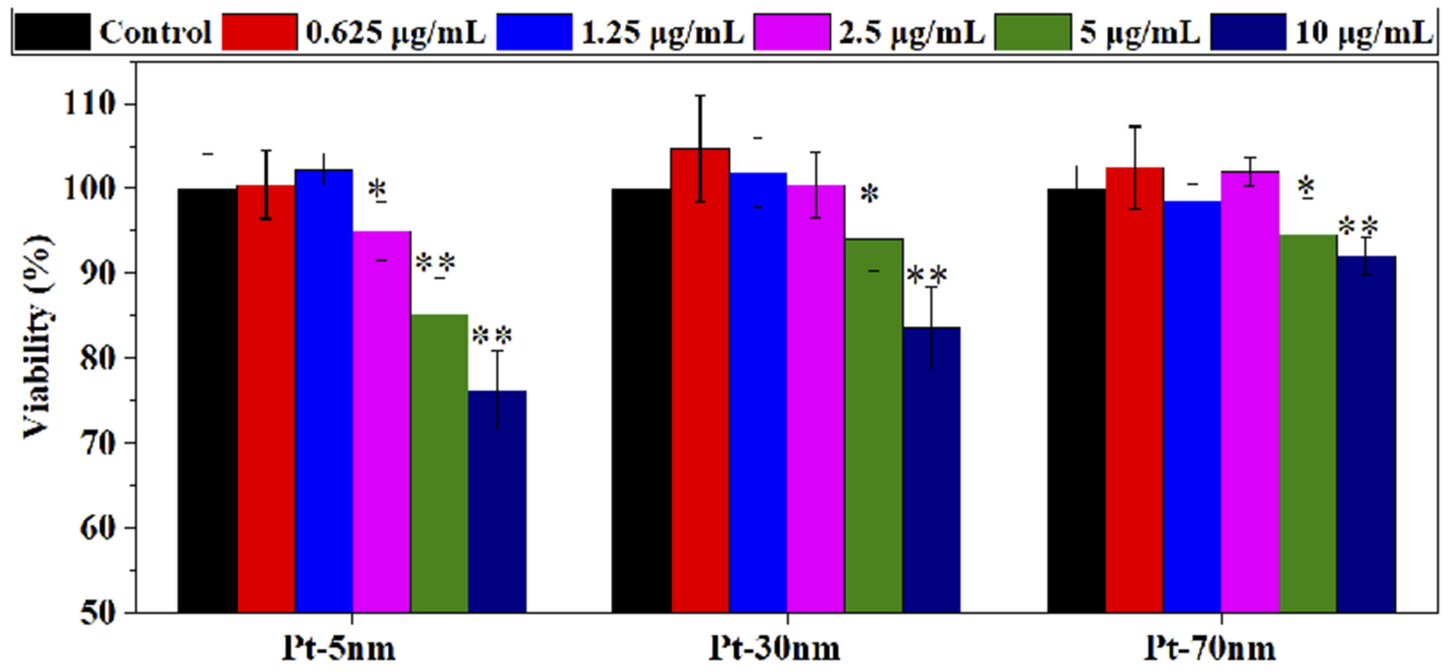

B

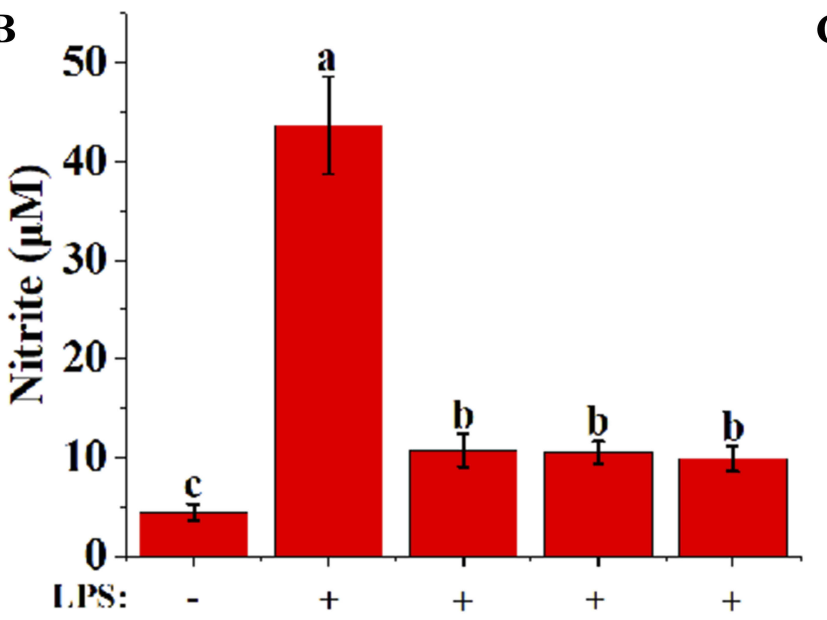

C

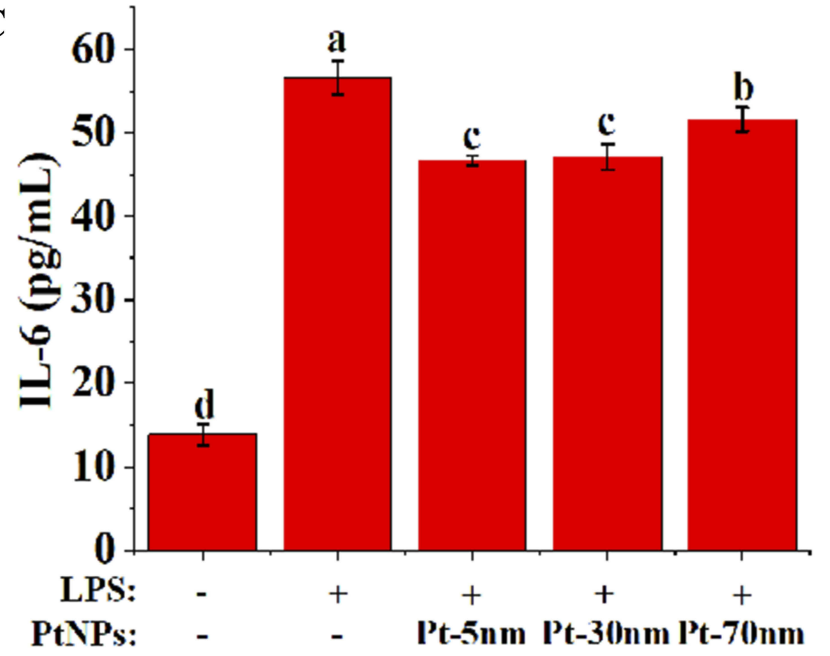

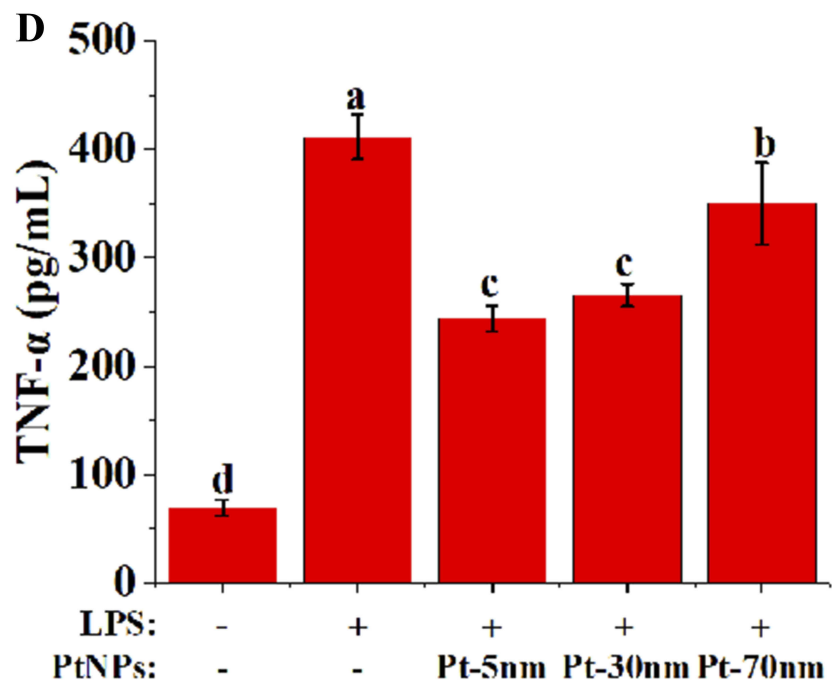

E 2000

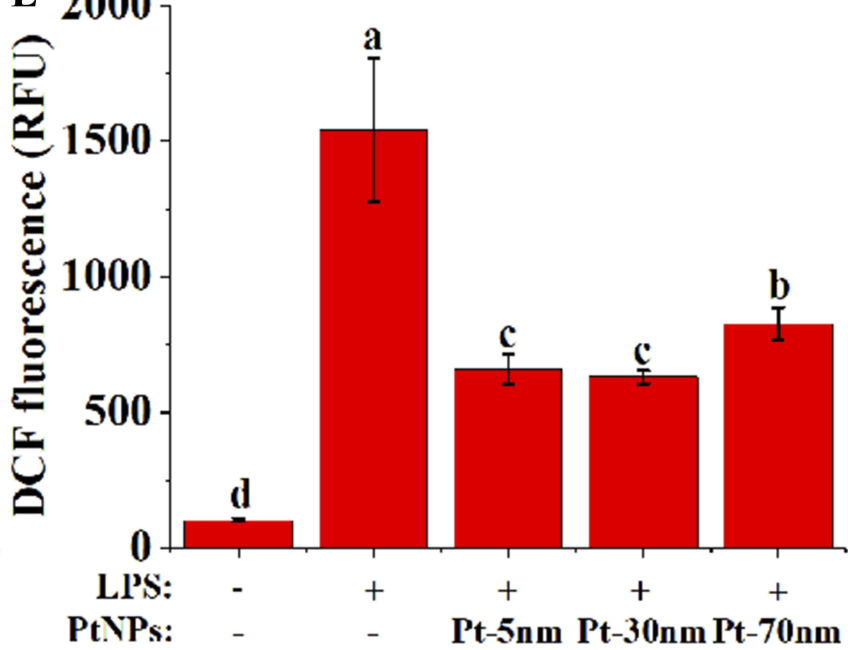

Figure 6 The anti-inflammatory effects of PtNPs in LPS-activated RAW264.7 cells: $(\mathbf{A})$ cell viability $(n=6)$, with significance labeled by $* P<0.05$ and $* * P<0.01$; production of $(B)$ nitric oxide $(n=6),(C)$ interleukin-6 (IL-6) $(n=3),(D)$ tumor necrosis factor- $\boldsymbol{\alpha}($ TNF- $\alpha)(n=3)$ and $(E)$ dichlorodihydrofluorescein $($ DCF) fluorescence $(n=6)$, with significance labeled by different lower-case letters. Data were expressed as mean \pm standard deviations, and were compared by one-way analysis of variance with Turkey's honest significant difference test. 
A
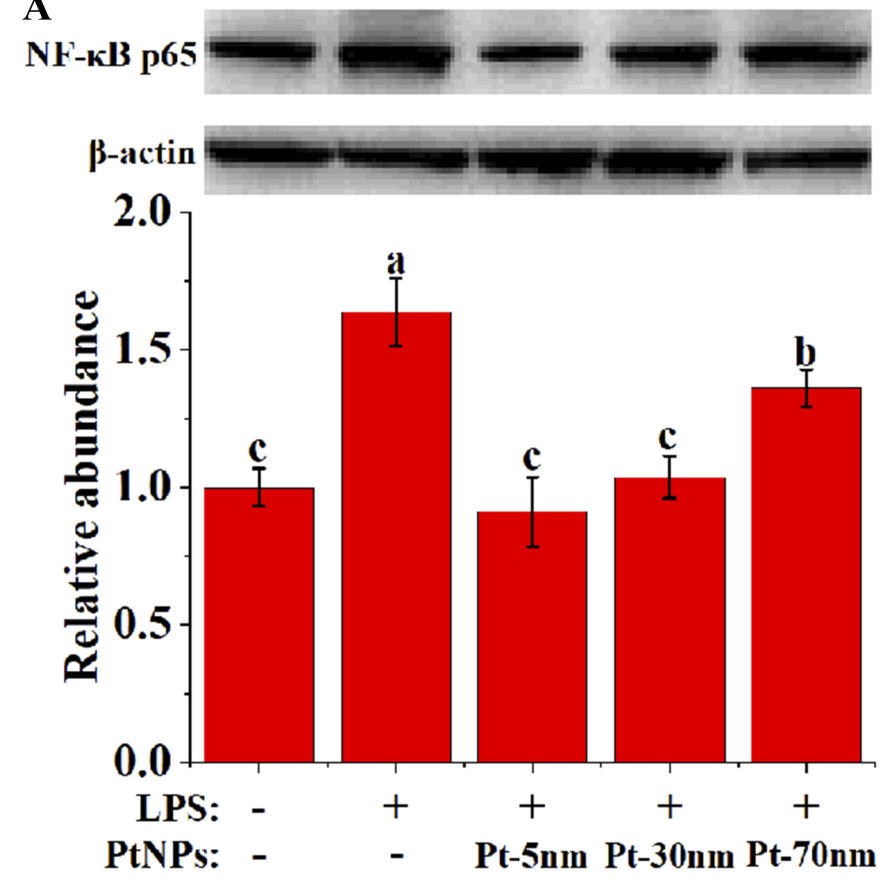

C
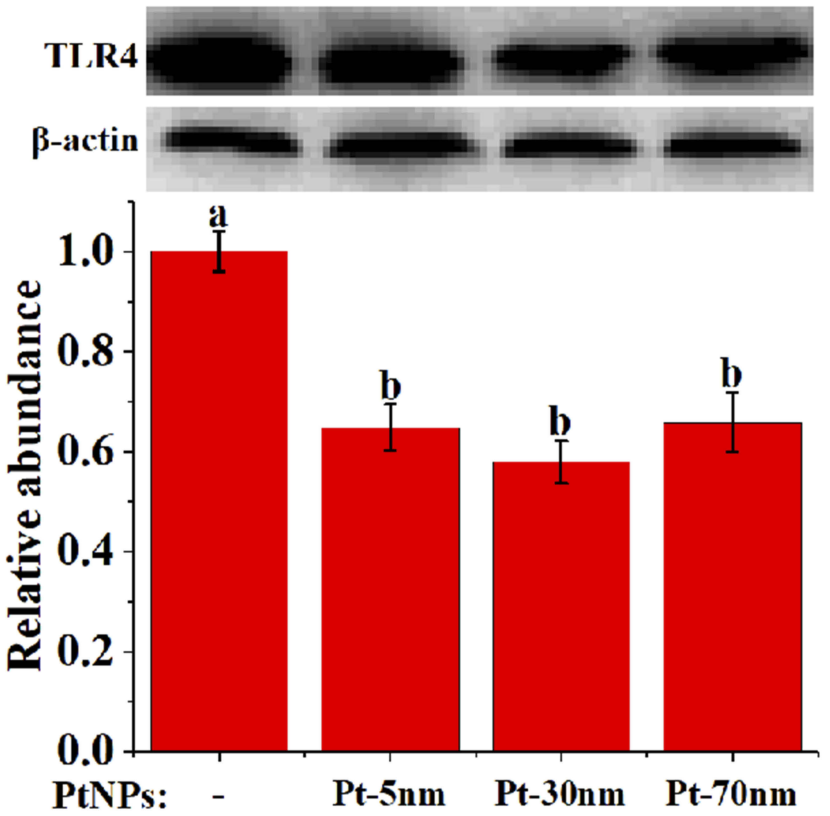

\section{B}
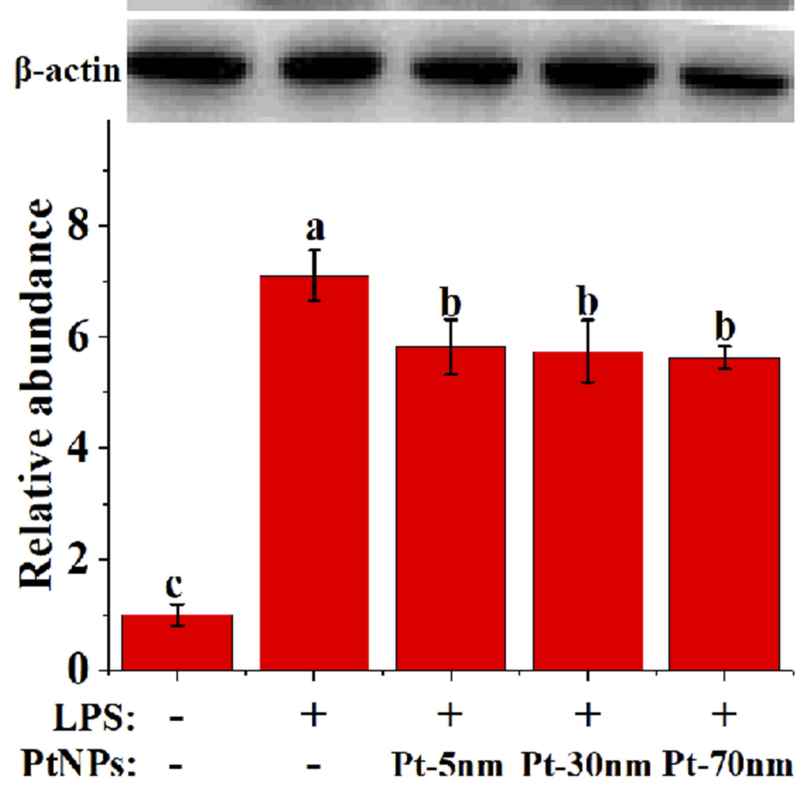

D
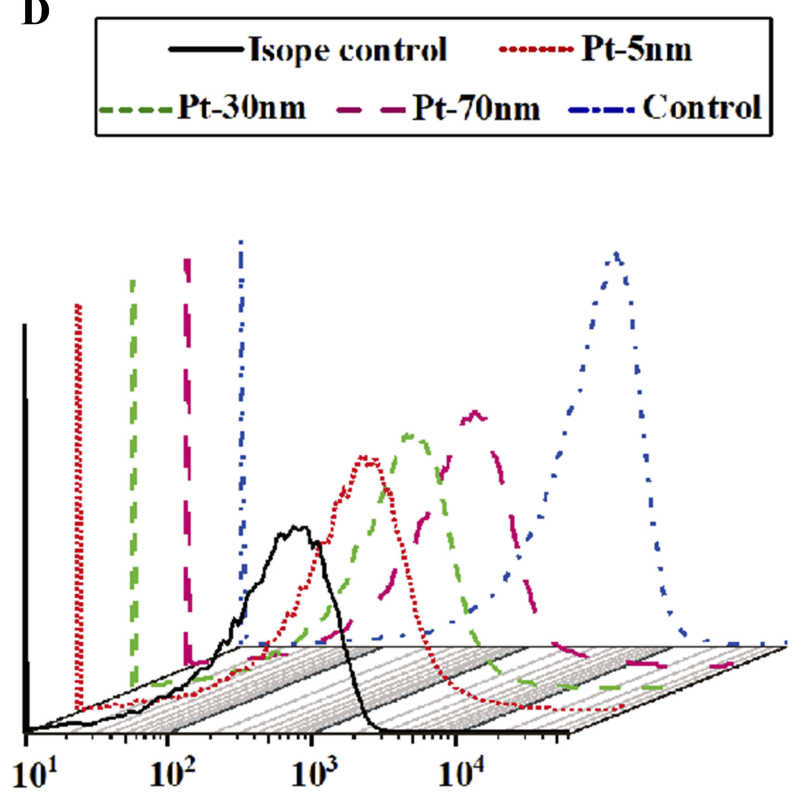

Figure 7 Analysis of the cell lysate levels of (A) nuclear factor kappa B (NF-kB) p65, (B) nitric oxide synthase (iNOS), and (C) Toll-like receptor 4 (TLR4) by Western blotting, and (D) the cell surface TLR4 by flow cytometry in RAW264.7 cells. Blots are representative of at least 3 replicates, and experiments were performed in triplicate. Immunoblots for actin were performed for each membrane to normalize protein loading between samples. Densitometry results are given in relation to normal controls that were set to be 1.0 , with significance labeled by different lower-case letters $(P<0.05$, one-way analysis of variance with Turkey's honest significant difference test).

orally administered PtNPs resulted a reduction in the abundance of fecal microbiome phylotypes in mice. However, a reduced biodiversity of gut microbiota has been associated with IBD, ${ }^{27}$ so, from this perspective, orally administered PtNPs seem to confer no benefit to gut microbiota in DSS-induced colitis mice.
As shown in Figure 8B, principal component analysis using unweighted UniFrac distances was performed to evaluate the $\beta$-diversity (between-sample taxonomic diversity). A clear separation of the three groups revealed distinct community structures of gut microbiota among these groups, and gut microbiota in the DSS + 


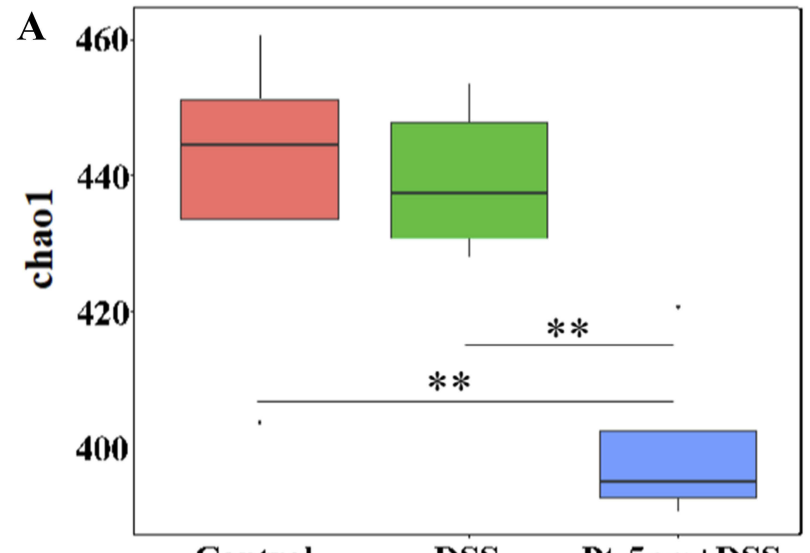

\section{D $\square$ DSS $\square$ Pt-5nm+DSS}

o_Pseudomonadales

g_Acinetobacter

f_Moraxellaceae

s_Acinetobacter_lwoffii

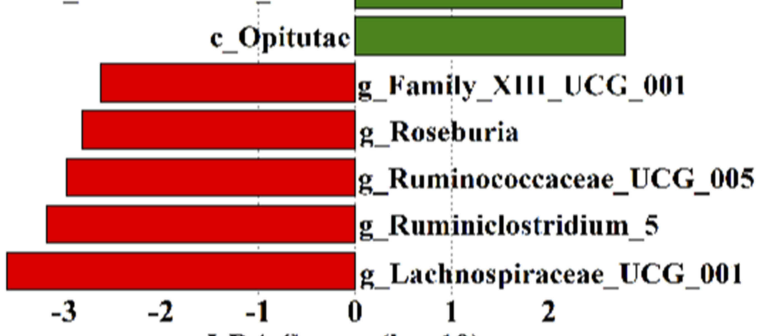

LDA Scores $(\log 10)$

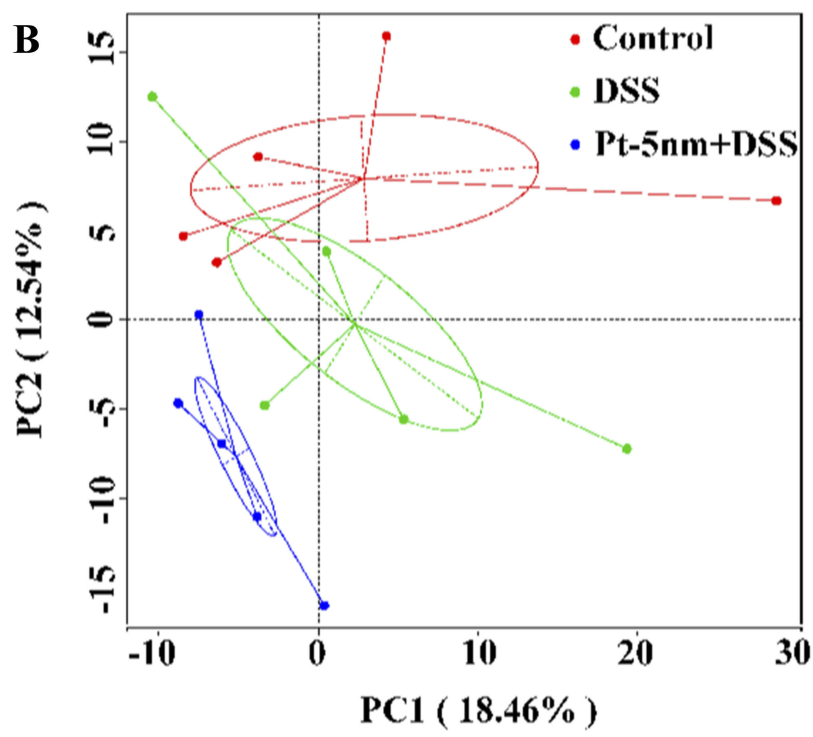

E Control $\square$ Pt-5nm+DSS

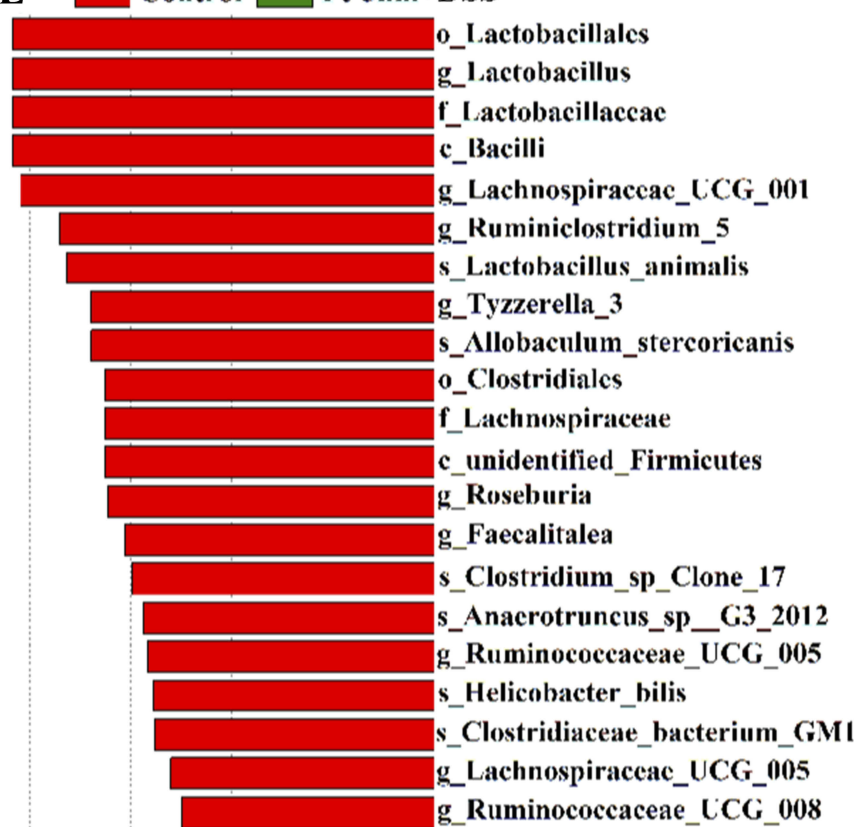

g_Acetatifactor

g_Acinetobacter

s_Acinetobacter_lwoffii

o_Pseudomonadales

f_Moraxellaceae

g_Klebsiella

c_ $\gamma$-proteobacteria

o_Enterobacteriales

f_Enterobacteriaceae g_Rikenella

$\begin{array}{cccccc}-2 & -1 & 0 & 1 & 2 & 3\end{array}$

Figure 8 The effects of PtNPs on gut-microbiota profile: (A) the Chaol metric for $\boldsymbol{\alpha}$-diversity; (B) unweighted principal component analysis of $\boldsymbol{\beta}$-diversity, with each point representing the result of one animal and the percentages indicating contribution of the principal components to sample difference; (C) the ratios of Firmicutes to Bacteroidetes; (D and E) linear discriminant analysis (LDA) scores of the differentially abundant taxa as identified by LDA effect size. The comparison in (A), (C), (D) and (E) was made by Wilcox's test with significance labeled by $* * P<0.0$ I or $* P<0.05$.

Pt-5 nm group seem to be phylogenetically closer to that in the DSS control group than that in the normal control group.
The proportions of two dominant phyla, Firmicutes and Bacteroidetes, in gut-microbiota correlate with diverse health status (e.g. aging, obese, diabetes, and autism), 
and depletion in Firmicutes or enrichment in Bacteroidetes has been linked to exacerbated inflammatory milieu in IBD. $^{26,28}$ The DSS + Pt-5nm group had a significantly lower level of Firmicutes/Bacteroidetes ratio than did the groups of DSS control $(P<0.05)$ and normal control $(P<0.05)$ (Figure $8 \mathrm{C})$, which implicates a potentially negative effect of PtNPs on the community structure of gut microbiota.

As revealed by the LEfSe algorithm (Figures S3, 8D and E), there were 6,10 , and 21 significantly different taxa (LDA score $>2$ ) at various levels of taxonomy between the groups of DSS control and normal control, DSS + Pt-5 nm and DSS control, and normal control and DSS + Pt $-5 \mathrm{~nm}$, respectively. In comparison with DSS control and normal control animals, the Pt-5 nm-treated mice showed an enrichment in the order Pseudomonales and the family Moraxellaceae (genus Acinetobacter), within the class $\gamma$-Proteobacteria that are overrepresented in both Crohn's disease and ulcerative colitis patients. ${ }^{29}$ In addition, in comparison with DSS control and normal control animals, oral administration of Pt-5 nm decreased several genera (e.g. Ruminococcaceae UCG-005, Roseburia, Lachnospiraceae UCG-001) within the families of Ruminococcaceae and Lachnospiraceae that are less abundant in Crohn's disease patients. ${ }^{30}$ These results again demonstrate a potential unfavorable effect of orally administered PtNPs on the gut microbial community.

Based on the above analysis of gut-microbiota profile, orally administered Pt-5 nm seemed to induce gut dysbiosis in mice. We thus propose that PtNPs exerted anti-colitis activities via their gut barrier-protecting and anti-inflammatory properties, rather than through gut-microbiota modulation.

\section{Discussion}

In DSS-induced mouse colitis, oral administration of PtNPs improved disease conditions including weight loss, DAI, colon shortening and colon histopathology. It also provided a marked protective effect against the leakage of intestinal epithelial barrier, which is recognized as the first step towards IBD. A reduction in colonic and systemic inflammation may serve as another underlying mechanism; while the in vitro cellular data support direct anti-inflammatory activities of PtNPs in macrophages through a mechanism involving intracellular ROS scavenging and TLR-4/NF- $\mathrm{kB}$ signaling suppression. Specifically, orally administered PtNPs, for the first time, were found to exert several potentially adverse effects on gut microbiota, which may raise a flag of caution for therapeutic applications of PtNPs via the oral route.

PtNPs have been reported to be nontoxic to intestinal epithelial cells at a concentration of up to $80 \mu \mathrm{g} / \mathrm{mL},{ }^{31}$ so PtNPs at the gavage concentration of $2.8 \mu \mathrm{g} / \mathrm{mL}$ in our animal experiment were unlikely to irritate intestinal epithelium. By damaging proteins, DNA and lipids, oxidative stress induces the pathogenesis of a myriad of diseases such as diabetes, neurodegeneration, atherosclerosis and cancer, while antioxidant supplementation has been recognized to exert favorable effects on disease control by restoring the redox balance. ${ }^{32}$ At the initial stage of IBD pathogenesis, mucosal injury induced by oxidative stress plays pivotal roles. ${ }^{33}$ In this study, PtNPs, for the first time, showed an evident gut-barrier protecting activity, which should be owning to their strong activity to catalyze hydrogen peroxide decomposition and to scavenge superoxide and singlet oxygen. ${ }^{10}$ In fact, PtNPs have also been found to attenuate oxidative injury in skin, brain, lung, and liver in the previous studies. ${ }^{13-15,34-36}$

ROS scavenging is generally regarded as the primary cytoprotective and anti-inflammatory mechanism of PtNPs in mammalian cell lines such as Caco-2, HeLa, HT-1080, RAW264.7 and THP-1. ${ }^{37-41}$ In line with Nomura et al. (2011) and Rehman et al (2012), ${ }^{41,42}$ PtNPs were observed to attenuate intracellular oxidant burst in RAW264.7 macrophages. The inhibitory effect of PtNPs on ROS production was found to be size-dependent, which aligns with the results of Chen et al. (2016) that smaller PtNPs possess greater enzyme-mimicking activities, ${ }^{43}$ and considering the proinflammatory role of intracellular ROS, ${ }^{25}$ this might be the reason for the size-dependent effects of PtNPs on cytokine production and NF- $\mathrm{KB}$ activation.

TLR4 protein reduction by PtNPs might be owning to co-endocytosis of membrane TLR4 with the particles, and has not been reported as an anti-inflammatory mechanism in macrophages before. The binding of LPS to cell surface TLR4 has been demonstrated to induce receptor-mediated endocytosis in human monocytes as a prompt mechanism of endotoxin desensitization. ${ }^{44}$ In Raw264.7 cells, the endocytic intake of gold nanoparticles has been found to cause a prompt phagosomal translocation of membrane TLR9, ${ }^{45}$ and has been found to be accelerated by LPS stimulation. ${ }^{46}$ We thus hypothesize that cellular uptake of PtNPs could result in co-endocytosis of membrane TLR4 leading to LPS desensitization, a new-found anti-inflammatory mechanism of PtNPs. 
Following their ingestion, nanoparticles pass through the digestive tract where they may meet and shape the gut microbiota. Recently, based on several animal and human studies, silver nanoparticles have been recognized for their toxicology on gut microbiota. ${ }^{47,48}$ Some other engineered nanoparticles frequently used for biomedical and food purposes, such as silica dioxide, zinc oxide, titanium dioxide, silver, carbon, gold and selenium nanoparticles, have also been implicated to exert positive or negative impacts on gut microbiota, when administered via an oral route. ${ }^{49-52}$ However, few studies have examined the interaction of PtNPs with gut microbiota. Here, orally administered PtNPs were found to reduce the phylotype richness and the Firmicutes/Bacteroidetes ratio, and to increase proinflammatory bacteria, which is similar to the results of gold and silver nanoparticles in previous studies. ${ }^{47,49} \mathrm{H}_{2} \mathrm{O}_{2}$ generation by host epithelial and innate immune system and commensal lactic acid bacteria contributes to the maintenance of gut-microbiota homeostasis, ${ }^{53}$ and therefore, we hypothesize that noble metal nanoparticles might induce gut dysbiosis by catalytic elimination of intraluminal $\mathrm{H}_{2} \mathrm{O}_{2}$.

\section{Conclusion}

In conclusion, our results report a considerable efficacy of PtNPs in maintaining gut health via attenuation of intestinal injury and inflammation, which might inspire future application of PtNPs as a novel therapeutic agent to treat IBD. However, probiotic or prebiotic supplementation might be needed in combination with PtNPs to avoid potential adverse effects of the particles on gut microbiota.

\section{Acknowledgments}

We thank Ms Yu Miao at the Affiliated Hospital of Qingdao University for measuring hematological parameters. This work was financially supported by the National Natural Science Foundation of China (No. 31601406).

\section{Disclosure}

The authors report no conflicts of interest in this work.

\section{References}

1. Kaplan GG. The global burden of IBD: from 2015 to 2025. Nature Reviews Gastroenterology and Hepatology. 2015;12(12):720-727. doi:10.1038/nrgastro.2015.150

2. Weimers P, Munkholm P. The natural history of IBD: lessons learned. Curr Treat Options Gastroenterol. 2018;16(1):101-111. doi:10.1007/ s11938-018-0173-3
3. Zhang Y-Z, Li -Y-Y. Inflammatory bowel disease: pathogenesis. World J Gastroenterol. 2014;20(1):91-99. doi:10.3748/wjg.v20.i1.91

4. Tian T, Wang ZL, Zhang JH. Pathomechanisms of oxidative stress in inflammatory bowel disease and potential antioxidant therapies. Oxid Med Cell Longev. 2017;2017:1-18. doi:10.1155/2017/4535194

5. Jacob JM, Rajan R, Aji M, Kurup GG, Pugazhendhi A. Bio-inspired $\mathrm{ZnS}$ quantum dots as efficient photo catalysts for the degradation of methylene blue in aqueous phase. Ceram Int. 2019;45(4):4857-4862. doi:10.1016/j.ceramint.2018.11.182

6. Vasantharaj S, Sathiyavimal S, Senthilkumar P, LewisOscar F, Pugazhendhi A. Biosynthesis of iron oxide nanoparticles using leaf extract of Ruellia tuberosa: antimicrobial properties and their applications in photocatalytic degradation. $J$ Photoch Photobio B. 2019;192:74-82. doi:10.1016/j.jphotobiol.2018.12.025

7. Pugazhendhi A, Edison TNJI, Karuppusamy I, Kathirvel B. Inorganic nanoparticles: a potential cancer therapy for human welfare. Int $J$ Pharm. 2018;539(1-2):104-111. doi:10.1016/j.ijpharm.2018.01.034

8. Kumar PSM, Thiripuranthagan S, Imai T, et al. Pt nanoparticles supported on mesoporous $\mathrm{CeO} 2$ nanostructures obtained through green approach for efficient catalytic performance toward ethanol electro-oxidation. Acs Sustain Chem Eng. 2017;5(12):11290-11299. doi:10.1021/acssuschemeng.7b02019

9. Ramkumar VS, Pugazhendhi A, Prakash S, et al. Synthesis of platinum nanoparticles using seaweed Padina gymnospora and their catalytic activity as PVP/PtNPs nanocomposite towards biological applications. Biomed Pharmacother. 2017;92:479-490. doi:10.1016/ j.biopha.2017.05.076

10. Liu Y, Wu HH, Li M, Yin JJ, Nie ZH. pH dependent catalytic activities of platinum nanoparticles with respect to the decomposition of hydrogen peroxide and scavenging of superoxide and singlet oxygen. Nanoscale. 2014;6(20):11904-11910. doi:10.1039/c4nr0 $3848 \mathrm{~g}$

11. Kumar PSM, Ponnusamy VK, Deepthi KR, et al. Controlled synthesis of Pt nanoparticle supported $\mathrm{TiO} 2$ nanorods as efficient and stable electrocatalysts for the oxygen reduction reaction. $J$ Mater Chem A. 2018;6(46):23435-23444. doi:10.1039/C8TA07380E

12. Yu X, Yuan L, Zhu N, Wang K, Xia Y. Fabrication of antimicrobial curcumin stabilized platinum nanoparticles and their anti-liver fibrosis activity for potential use in nursing care. $J$ Photoch Photobio $B$. 2019;195:27-32. doi:10.1016/j.jphotobiol.2019.03.023

13. Onizawa S, Aoshiba K, Kajita M, Miyamoto Y, Nagai A. Platinum nanoparticle antioxidants inhibit pulmonary inflammation in mice exposed to cigarette smoke. Pulm Pharmacol Ther. 2009;22 (4):340-349. doi:10.1016/j.pupt.2008.12.015

14. Takamiya M, Miyamoto Y, Yamashita T, Deguchi K, Ohta Y, Abe K. Strong neuroprotection with a novel platinum nanoparticle against ischemic stroke- and tissue plasminogen activator-related brain damages in mice. Neuroscience. 2012;221:47-55. doi:10.1016/j. neuroscience.2012.06.060

15. Takamiya M, Miyamoto Y, Yamashita T, et al. Neurological and pathological improvements of cerebral infarction in mice with platinum nanoparticles. $J$ Neurosci Res. 2011;89(7):1125-1133. doi:10.10 $02 /$ jnr.22622

16. Katao K, Honma R, Kato S, Watanabe S, Imai J. Influence of platinum nanoparticles orally administered to rats evaluated by systemic gene expression profiling. Experimental Animals. 2011;60 (1):33-45. doi:10.1538/expanim.60.33

17. Yogesh B, Vineeta B, Rammesh N, Saili P. Biosynthesized platinum nanoparticles inhibit the proliferation of human lung-cancer cells in vitro and delay the growth of a human lung-tumor xenograft in vivo: -In vitro and in vivo anticancer activity of bio-Pt NPs. Journal of Pharmacopuncture. 2016;19(2):114-121. doi:10.3831/KPI.2016.19.012

18. Laroui H, Ingersoll SA, Liu HC, et al. Dextran Sodium Sulfate (DSS) induces colitis in mice by forming nano-lipocomplexes with mediumchain-length fatty acids in the colon. Plos One. 2012;7:3. doi:10.13 71/journal.pone. 0032084 
19. Parasuraman S, Raveendran R, Kesavan R. Blood sample collection in small laboratory animals. J Pharmacol Pharmacother. 2010;1 (2):87-93. doi:10.4103/0976-500X.72350

20. Rodriguez-Palacios A, Aladyshkina N, Cominelli F. Stereomicroscopy and 3D-target myeloperoxidase intestinal phenotyping following a fecal flora homogenization protocol. Protocol Exchange. 2015. doi:10.1038/protex.2015.065

21. Chang J, Leong RW, Wasinger VC, Ip M, Yang M, Phan TG. Impaired intestinal permeability contributes to ongoing bowel symptoms in patients with inflammatory bowel disease and mucosal healing. Gastroenterology. 2017;153(3):723-+. doi:10.1053/j.gastro.2017.05.056

22. Ikwegbue PC, Masamba P, Oyinloye BE, Kappo AP. Roles of heat shock proteins in apoptosis, oxidative stress, human inflammatory diseases, and cancer. Pharmaceuticals (Basel). 2017;11:1. doi:10.33 90/ph11010002

23. Takahashi S, Andreoletti G, Chen R, et al. De novo and rare mutations in the HSPA1L heat shock gene associated with inflammatory bowel disease. Genome Med. 2017;9(1):8. doi:10.1186/s13073-016-0394-9

24. Gasche C, Lomer MCE, Cavill I, Weiss G. Iron, anaemia, and inflammatory bowel diseases. Gut. 2004;53(8):1190-1197. doi:10.11 36/gut.2003.035758

25. Morgan MJ, Liu ZG. Crosstalk of reactive oxygen species and NFkappa B signaling. Cell Res. 2011;21(1):103-115. doi:10.1038/cr.20 10.178

26. Clemente JC, Manasson J, Scher JU. The role of the gut microbiome in systemic inflammatory disease. BMJ. 2018;360:j5145. doi:10.11 36/bmj.j5145

27. Kostic AD, Xavier RJ, Gevers D. The microbiome in inflammatory bowel disease: current status and the future ahead. Gastroenterology 2014;146(6):1489-1499. doi:10.1053/j.gastro.2014.02.009

28. Clemente JC, Ursell LK, Parfrey LW, Knight R. The impact of the gut microbiota on human health: an integrative view. Cell. 2012;148 (6):1258-1270. doi:10.1016/j.cell.2012.01.035

29. Morgan XC, Tickle TL, Sokol H, et al. Dysfunction of the intestinal microbiome in inflammatory bowel disease and treatment. Genome Biol. 2012;13(9):R79. doi:10.1186/gb-2012-13-9-r79

30. Kaakoush NO, Day AS, Huinao KD, et al. Microbial dysbiosis in pediatric patients with Crohn's disease. J Clin Microbiol. 2012;50 (10):3258-3266. doi:10.1128/JCM.01396-12

31. Tuncer S, Colakoglu M, Ulusan S, Ertas G, Karasu C, Banerjee S. Evaluation of colloidal platinum on cytotoxicity, oxidative stress and barrier permeability across the gut epithelium. Heliyon. 2019;5(3): e01336. doi:10.1016/j.heliyon.2019.e01336

32. Liu ZW, Ren ZP, Zhang J, et al. Role of ROS and nutritional antioxidants in human diseases. Front Physiol. 2018;9:477.

33. Piechota-Polanczyk A, Fichna J. Review article: the role of oxidative stress in pathogenesis and treatment of inflammatory bowel diseases. Naunyn-Schmiedeberg's Arch Pharmacol. 2014;387(7):605-620. doi:10.1007/s00210-014-0985-1

34. Katsumi H, Fukui K, Sato K, et al. Pharmacokinetics and preventive effects of platinum nanoparticles as reactive oxygen species scavengers on hepatic ischemia/reperfusion injury in mice. Metallomics. 2014;6(5):1050-1056. doi:10.1039/c4mt00018h

35. Shibuya S, Ozawa Y, Watanabe K, et al. Palladium and platinum nanoparticles attenuate aging-like skin atrophy via antioxidant activity in mice. PLoS One. 2014;9:10. doi:10.1371/journal.pone.0109288

36. Yoshihisa Y, Honda A, Zhao QL, et al. Protective effects of platinum nanoparticles against UV-light-induced epidermal inflammation. Exp Dermatol. 2010;19(11):1000-1006. doi:10.1111/j.1600-0625.2010. 01128.x

37. Hamasaki T, Kashiwagi T, Imada T, et al. Kinetic analysis of superoxide anion radical-scavenging and hydroxyl radical-scavenging activities of platinum nanoparticles. Langmuir. 2008;24(14):7354 7364. doi:10.1021/1a704046f
38. Clark A, Zhu AP, Sun K, Petty HR. Cerium oxide and platinum nanoparticles protect cells from oxidant-mediated apoptosis. $J$ Nanopart Res. 2011;13(10):5547-5555. doi:10.1007/s11051-0110544-3

39. Zhang LB, Laug L, Munchgesang W, et al. Reducing stress on cells with apoferritin-encapsulated platinum nanoparticles. Nano Lett. 2010;10(1):219-223. doi:10.1021/n1903313r

40. Gatto F, Moglianetti M, Pompa PP, Bardi G. Platinum nanoparticles decrease reactive oxygen species and modulate gene expression without alteration of immune responses in THP-1 monocytes. Nanomaterials-Basel. 2018;8:6.

41. Rehman MU, Yoshihisa Y, Miyamoto Y, Shimizu T. The anti-inflammatory effects of platinum nanoparticles on the lipopolysaccharideinduced inflammatory response in RAW 264.7 macrophages. Inflammation Research. 2012;61(11):1177-1185. doi:10.1007/s000 11-012-0512-0

42. Nomura M, Yoshimura Y, Kikuiri T, et al. Platinum nanoparticles suppress osteoclastogenesis through scavenging of reactive oxygen species produced in RAW264.7 cells. J Pharmacol Sci. 2011;117 (4):243-252. doi:10.1254/jphs.11099fp

43. Chen $\mathrm{C}$, Fan $\mathrm{SH}, \mathrm{Li} \mathrm{C}$, et al. Platinum nanoparticles inhibit antioxidant effects of vitamin $\mathrm{C}$ via ascorbate oxidase-mimetic activity. $J$ Mater Chem B. 2016;4(48):7895-7901. doi:10.1039/C6TB02382G

44. Husebye H, Halaas O, Stenmark H, et al. Endocytic pathways regulate Toll-like receptor 4 signaling and link innate and adaptive immunity. Embo J. 2006;25(4):683-692. doi:10.1038/sj.emboj.760 0991

45. Tsai CY, Lu SL, Hu CW, Yeh CS, Lee GB, Lei HY. Size-dependent attenuation of TLR9 signaling by gold nanoparticles in macrophages. J Immunol. 2012;188(1):68-76. doi:10.4049/jimmunol.1100344

46. Liu ZM, Li WQ, Wang F, et al. Enhancement of lipopolysaccharideinduced nitric oxide and interleukin- 6 production by PEGylated gold nanoparticles in RAW264.7 cells. Nanoscale. 2012;4(22):7135-7142. doi: $10.1039 / \mathrm{c} 2 \mathrm{nr} 31355 \mathrm{c}$

47. Dahiya DK, Renuka PAK. Impact of nanosilver on gut microbiota: a vulnerable link. Future Microbiol. 2018;13(4):483-492. doi:10.2217/ fmb-2017-0103

48. Mercier-Bonin M, Despax B, Raynaud P, Houdeau E, Thomas M. Mucus and microbiota as emerging players in gut nanotoxicology: the example of dietary silver and titanium dioxide nanoparticles. Crit Rev Food Sci. 2018;58(6):1023-1032. doi:10.1080/10408398.2016. 1243088

49. Zhu SQ, Jiang XM, Boudreau MD, et al. Orally administered gold nanoparticles protect against colitis by attenuating Toll-like receptor 4-and reactive oxygen/nitrogen species-mediated inflammatory responses but could induce gut dysbiosis in mice. Journal of Nanobiotechnology. 2018;16:86.

50. Chen H, Zhao R, Wang B, et al. The effects of orally administered $\mathrm{Ag}, \mathrm{TiO} 2$ and $\mathrm{SiO} 2$ nanoparticles on gut microbiota composition and colitis induction in mice. NanoImpact. 2017;8:80-88. doi:10.1016/j. impact.2017.07.005

51. Li J, Lei RH, Li X, et al. The antihyperlipidemic effects of fullerenol nanoparticles via adjusting the gut microbiota in vivo. Part Fibre Toxicol. 2018;15:5.

52. Gangadoo S, Dinev I, Chapman J, et al. Selenium nanoparticles in poultry feed modify gut microbiota and increase abundance of Faecalibacterium prausnitzii. Appl Microbiol Biotechnol. 2018;102 (3):1455-1466. doi:10.1007/s00253-017-8688-4

53. Knaus UG, Hertzberger R, Pircalabioru GG, Yousefi SPM, dos Santos FB. Pathogen control at the intestinal mucosa - $\mathrm{H} 2 \mathrm{O} 2$ to the rescue. Gut Microbes. 2017;8(1):67-74. doi:10.1080/19490976.2017. 1279378 


\section{Publish your work in this journal}

The International Journal of Nanomedicine is an international, peerreviewed journal focusing on the application of nanotechnology in diagnostics, therapeutics, and drug delivery systems throughout the biomedical field. This journal is indexed on PubMed Central, MedLine, CAS, SciSearch ${ }^{\circledR}$, Current Contents ${ }^{\circledR} /$ Clinical Medicine,
Journal Citation Reports/Science Edition, EMBase, Scopus and the Elsevier Bibliographic databases. The manuscript management system is completely online and includes a very quick and fair peer-review system, which is all easy to use. Visit http://www.dovepress.com/ testimonials.php to read real quotes from published authors. 